Review Article

\title{
Particle Production in Ultrarelativistic Heavy-Ion Collisions: A Statistical-Thermal Model Review
}

\author{
S. K. Tiwari and C. P. Singh \\ Department of Physics, Banaras Hindu University, Varanasi 221005, India \\ Correspondence should be addressed to S. K. Tiwari; sktiwari4bhu@gmail.com
}

Received 13 June 2013; Accepted 23 August 2013

Academic Editor: Bhartendu K. Singh

Copyright (C) 2013 S. K. Tiwari and C. P. Singh. This is an open access article distributed under the Creative Commons Attribution License, which permits unrestricted use, distribution, and reproduction in any medium, provided the original work is properly cited.

\begin{abstract}
The current status of various thermal and statistical descriptions of particle production in the ultrarelativistic heavy-ion collisions experiments is presented in detail. We discuss the formulation of various types of thermal models of a hot and dense hadron gas (HG) and the methods incorporated in the implementing of the interactions between hadrons. It includes our new excluded-volume model which is thermodynamically consistent. The results of the above models together with the experimental results for various ratios of the produced hadrons are compared. We derive some new universal conditions emerging at the chemical freeze-out of HG fireball showing independence with respect to the energy as well as the structure of the nuclei used in the collision. Further, we calculate various transport properties of HG such as the ratio of shear viscosity-to-entropy using our thermal model and compare with the results of other models. We also show the rapidity as well as transverse mass spectra of various hadrons in the thermal HG model in order to outline the presence of flow in the fluid formed in the collision. The purpose of this review article is to organize and summarize the experimental data obtained in various experiments with heavy-ion collisions and then to examine and analyze them using thermal models so that a firm conclusion regarding the formation of quark-gluon plasma (QGP) can be obtained.
\end{abstract}

\section{Introduction}

One of the main purposes of various heavy-ion collision programmes running at various places such as relativistic heavy-ion collider (RHIC) at Brookhaven National Laboratory (BNL) and large hadron collider (LHC) at CERN is to understand the properties of strongly interacting matter and to study the possibility of a phase transition from a confined hot, dense hadron gas (HG) phase to a deconfined and/or chiral symmetric phase of quark matter called quark-gluon plasma (QGP) [1-9]. By colliding heavy-ions at ultrarelativistic energies, such a phase transition is expected to materialize and QGP can be formed in the laboratory. Unfortunately, the detection of the QGP phase is still regarded as an uphill task. However, the existence of a new form of a matter called strongly interacting QGP (sQGP) has been demonstrated experimentally [10]. There is much compelling evidence, for example, elliptic flow, high energy densities, and very low viscosity [11]. However, we do not have supportive evidence that this fluid is associated with the properties quark deconfinement and/or chiral symmetry restoration which are considered as direct indications for QGP formation [11]. Although various experimental probes have been devised, but a clean unambiguous signal has not yet been outlined in the laboratory. So our prime need at present is to propose some signals to be used for the detection of QGP. However for this purpose, understanding the behaviour and the properties of the background HG is quite essential because if QGP is not formed, matter will continue to exist in the hot and dense HG phase. In the ultrarelativistic nucleus-nucleus collisions, a hot and dense matter is formed over an extended region for a very brief time, and it is often called a "fireball". The quark matter in the fireball after subsequent expansion and cooling will be finally converted into HG phase. Recently, the studies of the transverse momentum spectra of dileptons [12-21] and hadrons [22-27] are used to deduce valuable information regarding temperature and energy density of the fireball. The schematic diagram for the conjectured space-time evolution of the fireball formed in the heavy-ion collisions is shown in Figure 1 [28]. The space-time evolution consists of four different stages as follows. (i) In the initial stage of collisions, 


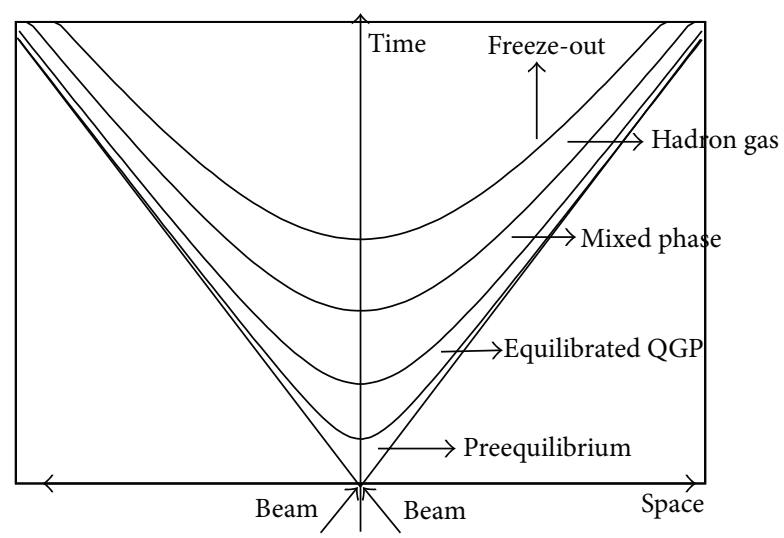

FIGURE 1: A schematic diagram of space-time evolution of ultrarelativistic heavy-ion collisions.

labeled as "Preequilibrium" in Figure 1, processes of partonparton hard scatterings may predominantly occur in the overlap region of two colliding nuclei, thus depositing a large amount of energy in the medium. The matter is still not in thermal equilibrium, and perturbative QCD models can describe the underlying dynamics as a cascade of freely colliding partons. The time of the preequilibrium state is predicted to about $1 \mathrm{fm} / \mathrm{c}$ or less. (ii) After the short preequilibrium stage, the QGP phase would be formed, in which partonparton and/or string-string interactions quickly contribute to attain thermal equilibrium in the medium. The energy density of this state is expected to reach above $3-5 \mathrm{GeV} / \mathrm{fm}^{3}$, equivalent to the temperature of $200-300 \mathrm{MeV}$. The volume then rapidly expands, and matter cools down. (iii) If the first order phase transition is assumed, the "mixed phase" is expected to exist between the QGP and hadron phases, in which quarks and gluons are again confined into hadrons at the critical temperature $T_{c}$. In the mixed phase, the entropy density is transferred into lower degrees of freedom, and therefore the system is prevented from a fast expansion. This leads to a maximum value in the lifetime of the mixed phase which is expected to last for a relatively long time $(\tau>10 \mathrm{fm} / \mathrm{c})$. (iv) In the hadronic phase, the system keeps collective expansion via hadron-hadron interactions, decreasing its temperature. Then, the hadronic interactions freeze after the system reaches a certain size and temperature, and hadrons that freely stream out from the medium are to be detected. There are two types of freeze-out stages. When inelastic collisions between constituents of the fireball do not occur any longer, we call this a chemical freeze-out stage. Later when the elastic collisions also cease to happen in the fireball, this stage specifies the thermal freeze-out.

Since many experiments running at various places measure the multiplicity, ratios, and so forth of various hadrons, it is necessary to know to which extent the measured hadron yields indicate equilibration. The level of equilibration of particles produced in these experiments is tested by analyzing the particle abundances $[22,23,29-57]$ or their momentum spectra $[22-27,37,38,46,47,58]$ using thermal models. Now, in the first case, one establishes the chemical composition of the system, while in the second case, additional information on dynamical evolution and collective flow can be extracted. Furthermore, study of the variations of multiplicity of produced particles with respect to collision energy, the momentum spectra of particles, and ratios of various particles have led to perhaps one of the most remarkable results corresponding to high energy strong interaction physics [6].

Recently various approaches have been proposed for the precise formulation of a proper equation of state (EOS) for hot and dense HG. Lacking lattice QCD results for the EOS at finite baryon density $n_{B}$, a common approach is to construct a phenomenological EOS for both phases of strongly interacting matter. Among those approaches, thermal models are widely used and indeed are very successful in describing various features of the HG. These models are based on the assumption of thermal equilibrium reached in HG. A simple form of the thermal model of hadrons is the ideal hadron gas (IHG) model in which hadrons and resonances are treated as pointlike and noninteracting particles. The introduction of resonances in the system is expected to account for the existence of attractive interactions among hadrons [59]. But in order to account for the realistic behaviour of HG, a short range repulsion must also be introduced. The importance of such correction is more obvious when we calculate the phase transition using IHG picture which shows the reappearance of hadronic phase as a stable configuration in the simple Gibbs construction of the phase equilibrium between the HG and QGP phases at very high baryon densities or chemical potentials. This anomalous situation [60-62] cannot be explained because we know that the stable phase at any given $\left(T, \mu_{B}\right)$ is the phase which has a larger pressure. Once the system makes a transition to the QGP phase, it is expected to remain in that phase even at extremely large $T$ and $\mu_{B}$ due to the property of asymptotic freedom of QCD. Moreover, it is expected that the hadronic interactions become significant when hadrons are densely packed in a hot and dense hadron gas. One significant way to handle the repulsive interaction between hadrons is based on a macroscopic description in which the hadrons are given a geometrical size, and hence they will experience a hardcore repulsive interaction when they touch each other, and consequently a van-der Waals excluded-volume effect is visible. As a result, the particle yields are essentially reduced in comparison to that of IHG model, and also the anomalous situation in the phase transition mentioned above disappears. Recently, many phenomenological models incorporating excluded-volume effect have been widely used to account for the properties of hot and dense HG [63-76]. However, these descriptions usually suffer from some serious shortcomings. First, mostly, the descriptions are thermodynamically inconsistent because one does not have a well-defined partition function or thermodynamical potential $(\Omega)$ from which other thermodynamical quantities can be derived, for example, the baryon density $\left(n_{B}\right) \neq \partial \Omega / \partial \mu_{B}$. Secondly, for the dense hadron gas, the excluded-volume model violates causality (i.e., velocity of sound $c_{s}$ in the medium is greater than the velocity of light). So, although some of the models explain the data very well, such shortcomings make the models mostly unattractive. Sun et al. [76] have incorporated the effect of relativistic correction in the formulation of an EOS for HG. However, 
such effect is expected to be very small because the masses of almost all the hadrons present in the hot and dense HG are larger than the temperature of the system; so they are usually treated as nonrelativistic particles except pion whose mass is comparable to the temperature, but most of the pions come from resonances whose masses are again larger than the temperature of HG [77]. In [78], two-source thermal model of an ideal hadron gas is used to analyze the experimental data on hadron yields and ratios. In this model, the two sources, a central core and a surrounding halo, are in local equilibrium at chemical freeze-out. It has been claimed that the excludedvolume effect becomes less important in the formulation of EOS for hadron gas in the two-source model.

Another important approach used in the formulation of an EOS for the HG phase is mean-field theoretical models [79-82] and their phenomenological generalizations [8385]. These models use the local renormalizable Lagrangian densities with baryonic and mesonic degrees of freedom for the description of HG phase. These models rigorously respect causality. Most importantly they also reproduce the ground state properties of the nuclear matter in the low-density limit. The short-range repulsive interaction in these models arises mainly due to $\omega$-exchange between a pair of baryons. It leads to the Yukawa potential $V(r)=\left(G^{2} / 4 \pi r\right) \exp \left(-m_{\omega} r\right)$, which further gives mean potential energy as $U_{B}=G^{2} n_{B} / m_{\omega}$. It means that $U_{B}$ is proportional to the net baryon density $n_{B}$. Thus $U_{B}$ vanishes in the $n_{B} \rightarrow 0$ limit. In the baryonless limit, hadrons (mesons) can still approach pointlike behaviour due to the vanishing of the repulsive interactions between them. It means that, in principle, one can excite a large number of hadronic resonances at large $T$. This will again make the pressure in the HG phase larger than the pressure in the QGP phase, and the hadronic phase would again become stable at sufficiently high temperature, and the Gibbs construction can again yield HG phase at large $T$. In some recent approaches this problem has been cured by considering another temperature-dependent mean-field $U_{\mathrm{VDW}}(n, T)$, where $n$ is the sum of particle and antiparticle number densities. Here $U_{\mathrm{VDW}}(n, T)$ represents van-der Waals hardcore repulsive interaction between two particles and depends on the total number density $n$ which is nonzero even when net baryon density $n_{B}$ is zero in the large temperature limit $[72,73,75]$. However, in the high-density limit, the presence of a large number of hyperons and their unknown couplings to the mesonic fields generates a large amount of uncertainty in the EOS of HG in the mean-field approach. Moreover, the assumption of how many particles and their resonances should be incorporated in the system is a crucial one in the formulation of EOS in this approach. The meanfield models can usually handle very few resonances only in the description of HG and hence are not as such reliable ones [75].

In this review, we discuss the formulation of various thermal models existing in the literature quite in detail and their applications to the analysis of particle production in ultrarelativistic heavy-ion collisions. We show that it is important to incorporate the interactions between hadrons in a consistent manner while formulating the EOS for hot, dense HG. For repulsive interactions, van-der Waals type of excluded-volume effect is often used in thermal models, while resonances are included in the system to account for the attractive interactions. We precisely demand that such interactions must be incorporated in the models in a thermodynamically consistent way. There are still some thermal models in the literature which lack thermodynamical self-consistency. We have proposed a new excluded-volume model where an equal hardcore size is assigned to each type of baryons in the HG, while the mesons are treated as pointlike particles. We have successfully used this model in calculating various properties of $\mathrm{HG}$ such as number density, and energy density. We have compared our results with those of the other models. Further, we have extracted chemical freeze-out parameters in various thermal models by analyzing the particle ratios over a broad energy range and parameterized them with the center-of-mass energy. We use these parameterizations to calculate the particle ratios at various center-of-mass energies and compare them with the experimental data. We further provide a proposal in the form of freeze-out conditions for a unified description of chemical freeze-out of hadrons in various thermal models. An extension of the thermal model for the study of the various transport properties of hadrons will also be discussed. We also analyze the rapidity as well as transverse mass spectra of hadrons using our thermal model and examine the role of any flow existing in the medium by matching the theoretical results with the experimental data. Thus the thermal approach indeed provides a very satisfactory description of various features of HG by reproducing a large number of experimental results covering wide energy range from alternating gradient synchrotron (AGS) to large hadron collider (LHC) energy.

\section{Formulation of Thermal Models}

Various types of thermal models for HG using excludedvolume correction based on van-der Waals type effect have been proposed. Thermal models have often used the grand canonical ensemble description to write the partition function for the system because it suits well for systems with large number of produced hadrons [30] and/or large volume. However, for nonrelativistic statistical mechanics, the use of a grand canonical ensemble is usually just a matter of convenience [86]. Furthermore, the canonical ensemble can be used in case of small systems (e.g., peripheral nucleusnucleus collisions) and for low energies in case of strangeness production $[87,88]$ due to canonical suppression of the phase space. Similarly some descriptions also employ isobaric partition function in the derivation of their HG model. We succinctly summarize the features of some models as follows.

2.1. Hagedorn Model. In the Hagedorn model $[67,68]$, it is assumed that the excluded-volume correction is proportional to the pointlike energy density $\epsilon^{0}$. It is also assumed that the density of states of the finite-size particles in total volume $V$ can be taken as precisely the same as that of pointlike particles in the available volume $\Delta$ where $\Delta=V-\Sigma_{i} V_{i}^{0}$ and $V_{i}^{0}$ is the 
eigen volume of the $i$ th particle in the HG. Thus, the grand canonical partition function satisfies the relation:

$$
\ln Z(T, V, \lambda)=\ln Z^{0}(T, \Delta, \lambda) .
$$

The sum of eigen volumes $\Sigma_{i} V_{i}^{0}$ is given by the ratio of the invariant cluster mass to the total energy density, and $\lambda$ is the fugacity, that is, $\lambda=\exp (\mu / T)$. Hence $\Sigma_{i} V_{i}^{0}=E / 4 B=$ $V \epsilon / 4 B$, and the energy density $\epsilon=\Delta \epsilon^{0} / V$. $\epsilon^{0}$ is the energy density when particles are treated as pointlike. Now, using the expression for $\Delta$, one finally gets

$$
\epsilon_{i}^{\mathrm{ex}}=\frac{\epsilon_{i}^{0}}{1+\epsilon^{0} / 4 B} .
$$

When $\epsilon^{0} / 4 B \gg 1, \epsilon=\Sigma_{i} \epsilon_{i}^{\text {ex }}=4 B$ which is obviously the upper limit for $\epsilon$ since it gives the energy density existing inside a nucleon and is usually regarded as the latent heat density required for the phase transition. Here $B$ represents the bag constant. The expressions of number density and pressure can similarly be written as follows:

$$
\begin{aligned}
& n_{i}^{\mathrm{ex}}=\frac{n_{i}^{0}}{1+\epsilon^{0} / 4 B}, \\
& P_{i}^{\mathrm{ex}}=\frac{P_{i}^{0}}{1+\epsilon^{0} / 4 B} .
\end{aligned}
$$

Here, $n_{i}^{0}$ and $P_{i}^{0}$ are the number density and pressure of pointlike particles, respectively.

2.2. Cleymans-Suhonen Model. In order to include van-der Waals type of repulsion between baryons, Cleymans and Suhonen [63] assigned an equal hardcore radius to each baryon. Consequently, the available volume for baryons is $V-\Sigma_{i} N_{i} V_{i}^{0}$; here $V_{i}^{0}$ is the eigen volume of $i$ th baryon, and $N_{i}$ is the total number. As a result, the net excluded number density, pressure, and the energy density of a multicomponent HG are given as follows:

$$
\begin{aligned}
n^{\mathrm{ex}} & =\frac{\sum_{i} n_{i}^{0}}{1+\sum_{i} n_{i}^{0} V_{i}^{0}}, \\
P^{\mathrm{ex}} & =\frac{\sum_{i} P_{i}^{0}}{1+\sum_{i} n_{i}^{0} V_{i}^{0}}, \\
\epsilon^{\mathrm{ex}} & =\frac{\sum_{i} \epsilon_{i}^{0}}{1+\sum_{i} n_{i}^{0} V_{i}^{0}},
\end{aligned}
$$

where $n_{i}^{0}, P_{i}^{0}$, and $\epsilon_{i}^{0}$ are net baryon density, pressure, and energy density of pointlike particles, respectively, and $\Sigma_{i} n_{i}^{0} V_{i}^{0}$ is the fraction of occupied volume. Kuono and Takagi [66] modified these expressions by considering the existence of a repulsive interaction either between a pair of baryons or between a pair of antibaryons only. Therefore, the expressions (4), (5), and (6) take the folowing forms:

$$
\begin{aligned}
n^{\mathrm{ex}} & =\frac{\sum_{i} n_{i}^{0}}{1+\sum_{i} n_{i}^{0} V_{i}^{0}}-\frac{\sum_{i} \bar{n}_{i}^{0}}{1+\sum_{n_{i}^{0}} V_{i}^{0}}+n_{M}^{0}, \\
P^{\mathrm{ex}} & =\frac{\sum_{i} P_{i}^{0}}{1+\sum_{i} n_{i}^{0} V_{i}^{0}}-\frac{\sum_{i} \bar{P}_{i}^{0}}{1+\sum_{i} \bar{n}_{i}^{0} V_{i}^{0}}+P_{M}^{0}, \\
\epsilon^{\mathrm{ex}} & =\frac{\sum_{i} \epsilon_{i}^{0}}{1+\sum_{i} n_{i}^{0} V_{i}^{0}}+\frac{\sum_{i} \bar{\epsilon}_{i}^{0}}{1+\sum_{i} \bar{n}_{i}^{0} V_{i}^{0}}+\epsilon_{M}^{0},
\end{aligned}
$$

where $n_{i}^{0}$ and $\bar{n}_{i}^{0}$ are the number density of the pointlike baryons and antibaryons, respectively and $\epsilon_{i}^{0}\left(\bar{\epsilon}_{i}^{0}\right)$ and $P_{i}^{0}\left(\bar{P}_{i}^{0}\right)$ are the corresponding energy density and pressure. Similarly, $n_{M}^{0}, P_{M}^{0}$, and $\epsilon_{M}^{0}$ are the number density, pressure, and energy density of pointlike mesons, respectively.

2.3. Rischke-Gorenstein-Stocker-Greiner (RGSG) Model. The above discussed models possess a shortcoming that they are thermodynamically inconsistent because the thermodynamical variables like $n_{B}$ cannot be derived from a partition function or thermodynamical potential $(\Omega)$, for example, $n_{B} \neq \partial \Omega / \partial \mu_{B}$. Several proposals have come to correct such types of inconsistencies. Rischke et al. [69] have attempted to obtain a thermodynamically consistent formulation. In this model, the grand canonical partition function $Z_{G}$ for pointlike baryons can be written in terms of canonical partition function $Z_{C}$ as follows:

$$
Z_{G}^{0}(T, \mu, V)=\sum_{N=0}^{\infty} \exp \left(\mu \frac{N}{T}\right) Z_{C}(T, N, V) .
$$

They further modified the canonical partition function $Z_{C}$ by introducing a step function in the volume so as to incorporate excluded-volume correction into the formalism. Therefore, the grand canonical partition function (8) finally takes the following form:

$$
\begin{aligned}
Z_{G}^{\mathrm{ex}}\left(T, \mu, V-V^{0} N\right) \\
=\sum_{N=0}^{\infty} \exp \left(\mu \frac{N}{T}\right) Z_{C}\left(T, N, V-V^{0} N\right) \\
\quad \times \theta\left(V-V^{0} N\right) .
\end{aligned}
$$

The above ansatz is motivated by considering $N$ particles with eigen-volume $V^{0}$ in a volume $V$ as $N$ pointlike particles in the available volume $V-V^{0} N$ [69]. But, the problem in the calculation of (9) is the dependence of the available volume on the varying number of particles $N$ [69]. To overcome this difficulty, one should use the Laplace transformation of (9). Using the Laplace transform, one gets the isobaric partition function as follows:

$$
Z_{P}=\int_{0}^{\infty} d V \exp (-\xi V) Z_{G}^{\mathrm{ex}}\left(T, \mu, V-V^{0} N\right),
$$


or

$$
Z_{P}=\int_{0}^{\infty} d x \exp \left\{-x\left[\xi-\frac{\ln Z_{G}^{0}(T, \tilde{\mu})}{x}\right]\right\},
$$

where $x=V-V^{0} N$ and $\tilde{\mu}=\mu-T V^{0} \xi$. Finally, we get a transcendental type of equation as follows:

$$
P^{\mathrm{ex}}(T, \mu)=P^{0}(T, \tilde{\mu})
$$

where,

$$
\widetilde{\mu}=\mu-V^{0} P^{\mathrm{ex}}(T, \mu) .
$$

The expressions for the number density, entropy density, and energy density in this model can thus take a familiar form like

$$
\begin{gathered}
n^{\mathrm{ex}}(T, \mu)=\frac{\partial P^{0}(T, \tilde{\mu})}{\partial \tilde{\mu}} \frac{\partial \tilde{\mu}}{\partial \mu}=\frac{n^{0}(T, \tilde{\mu})}{1+V^{0} n^{0}(T, \tilde{\mu})}, \\
s_{1}^{\mathrm{ex}}(T, \mu)=\frac{\partial P^{0}(T, \tilde{\mu})}{\partial T}=\frac{s_{1}^{0}(T, \tilde{\mu})}{1+V^{0} n^{0}(T, \tilde{\mu})}, \\
\epsilon^{\mathrm{ex}}(T, \mu)=\frac{\epsilon^{0}(T, \tilde{\mu})}{1+V^{0} n^{0}(T, \tilde{\mu})} .
\end{gathered}
$$

These equations resemble (4) and (6) as given in CleymansSuhonen model [63] with $\mu$ being replaced by $\tilde{\mu}$. The above model can be extended for a hadron gas involving several baryonic species as follows:

$$
P^{\mathrm{ex}}\left(T, \mu_{1}, \ldots, \mu_{h}\right)=\sum_{i=1}^{h} P_{i}^{0}\left(T, \tilde{\mu}_{i}\right),
$$

where,

$$
\tilde{\mu}_{i}=\mu_{i}-V_{i}^{0} P^{\mathrm{ex}}\left(T, \mu_{i}\right),
$$

with $i=1, \ldots, h$. Particle number density for the $i$ th species can be calculated from following equation:

$$
n_{i}^{\mathrm{ex}}\left(T, \mu_{i}\right)=\frac{n_{i}^{0}\left(T, \tilde{\mu}_{i}\right)}{1+\sum_{j=1}^{h} V_{j}^{0} n_{j}^{0}\left(T, \tilde{\mu}_{j}\right)} .
$$

Unfortunately, the above model involves cumbersome, transcendental expressions which are usually not easy to calculate. Furthermore, this model fails in the limit of $\mu_{B}=0$ because $\widetilde{\mu}_{B}$ becomes negative in this limit.

2.4. New Excluded-Volume Model. Singh et al. [70] have proposed a thermodynamically consistent excluded-volume model in which the grand canonical partition function using Boltzmann approximation can be written as follows:

$$
\begin{aligned}
\ln Z_{i}^{\mathrm{ex}}= & \frac{g_{i} \lambda_{i}}{6 \pi^{2} T} \int_{V_{i}^{0}}^{V-\sum_{j} N_{j} V_{j}^{0}} d V \\
& \times \int_{0}^{\infty} \frac{k^{4} d k}{\sqrt{k^{2}+m_{i}^{2}}} \exp \left(-\frac{\sqrt{k^{2}+m_{i}^{2}}}{T}\right),
\end{aligned}
$$

where $g_{i}$ and $\lambda_{i}=\exp \left(\mu_{i} / T\right)$ are the degeneracy factor and the fugacity of $i$ th species of baryons, respectively, $k$ is the magnitude of the momentum of baryons, and $V_{i}^{0}$ is the eigenvolume assigned to each baryon of $i$ th species; hence $\sum_{j} N_{j} V_{j}^{0}$ becomes the total occupied volume where $N_{j}$ represents the total number of baryons of $j$ th species. We can rewrite (18) as follows:

$$
\ln Z_{i}^{\mathrm{ex}}=V\left(1-\sum_{j} n_{j}^{\mathrm{ex}} V_{j}^{0}\right) I_{i} \lambda_{i}
$$

where integral $I_{i}$ is

$$
I_{i}=\frac{g_{i}}{2 \pi^{2}}\left(\frac{m_{i}}{T}\right)^{2} T^{3} K_{2}\left(\frac{m_{i}}{T}\right) .
$$

Thus we have obtained the grand canonical partition function as given by (19) by incorporating the excluded-volume effect explicitly in the partition function. The number density of baryons after excluded-volume correction $\left(n_{i}^{\mathrm{ex}}\right)$ can be obtained as

$$
n_{i}^{\mathrm{ex}}=\frac{\lambda_{i}}{V}\left(\frac{\partial \ln Z_{i}^{\mathrm{ex}}}{\partial \lambda_{i}}\right)_{T, V} .
$$

So our prescription is thermodynamically consistent, and it leads to a transcendental equation:

$$
n_{i}^{\mathrm{ex}}=(1-R) \lambda_{i} I_{i}-I_{i} \lambda_{i}^{2} \frac{\partial R}{\partial \lambda_{i}} .
$$

Here $R=\sum_{i} n_{i}^{\mathrm{ex}} V_{i}^{0}$ is the fractional occupied volume. It is clear that if we put the factor $\partial R / \partial \lambda_{i}=0$ and consider only one type of baryons in the system, then (22) can be reduced to the thermodynamically inconsistent expression (4). The presence of $\partial R / \partial \lambda_{i}$ in (22) thus removes the thermodynamical inconsistency. For single-component HG, the solution of (22) can be taken as follows:

$$
n^{\mathrm{ex}}=\frac{1}{V} \frac{\int_{0}^{\lambda} d \lambda \exp \left[-1 / I V^{0} \lambda\right]}{\lambda \exp \left[-1 / I V^{0} \lambda\right]} .
$$

For a multicomponent hadron gas, (22) takes the following form:

$$
R=(1-R) \sum_{i} I_{i} V_{i}^{0} \lambda_{i}-\sum_{i} I_{i} V_{i}^{0} \lambda_{i}^{2} \frac{\partial R}{\partial \lambda_{i}}
$$

Using the method of parametric space [89], we write

$$
\lambda_{i}(t)=\frac{1}{\left(a_{i}+I_{i} V_{i}^{0} t\right)},
$$

where $a_{i}$ is the parameter and $t$ gives the space. We finally get the solution of (24) as follows:

$$
R=1-\frac{\int_{t}^{\infty}\left[\exp \left(-t^{\prime}\right) / G\left(t^{\prime}\right)\right] d t^{\prime}}{\exp (-t) / G(t)}
$$


where $t$ is a parameter such that

$$
\begin{gathered}
\frac{d \lambda_{i}(t)}{d t}=-I_{i} \lambda_{i}^{2} V_{i}^{0}, \\
G(t)=t \prod_{i=2}^{h}\left(a_{i}+I_{i} V_{i}^{0} t\right) .
\end{gathered}
$$

If $\lambda_{i}$ 's and $t$ are known, one can determine $a_{i}$. The quantity $t$ is fixed by setting $a_{1}=0$, and one obtains $t=1 / I_{1} V_{1} \lambda_{1}$; here the subscript 1 denotes the nucleon degree of freedom, and $h$ is the total number of baryonic species. Hence by using $R$ and $\partial R / \partial \lambda_{i}$ one can calculate $n_{i}$. It is obvious that the above solution is not unique, since it contains some parameters such as $a_{i}$, one of which has been fixed to zero arbitrarily. Alternatively, one can assume that [70]

$$
\frac{\partial R}{\partial \lambda_{i}}=\frac{\partial \sum_{j} n_{j}^{\mathrm{ex}} V_{j}^{0}}{\partial \lambda_{i}}=\left(\frac{\partial n_{i}^{\mathrm{ex}}}{\partial \lambda_{i}}\right) V_{i}^{0} .
$$

Here an assumption is made that the number density of $i$ th baryon will only depend on the fugacity of same baryon. Then (24) reduces to a simple form as

$$
\frac{\partial n_{i}^{\mathrm{ex}}}{\partial \lambda_{i}}+n_{i}^{\mathrm{ex}}\left(\frac{1}{I_{i} V_{i}^{0} \lambda_{i}^{2}}+\frac{1}{\lambda_{i}}\right)=\frac{1}{\lambda_{i} V_{i}^{0}}\left(1-\sum_{i \neq j} n_{j}^{\mathrm{ex}} V_{j}^{0}\right) .
$$

The solution of (29) can be obtained in a straight forward manner as follows [70]:

$$
n_{i}^{\mathrm{ex}}=\frac{Q_{i}\left(1-\sum_{j \neq i} n_{j}^{\mathrm{ex}} V_{j}^{0}\right)}{\lambda_{i} V_{i}^{0}} \exp \left(\frac{1}{I_{i} V_{i}^{0} \lambda_{i}}\right)
$$

where

$$
Q_{i}=\int_{0}^{\lambda_{i}} \exp \left(-\frac{1}{I_{i} V_{i}^{0} \lambda_{i}}\right) d \lambda_{i}
$$

Now, $R$ can be obtained by using the following relation:

$$
R=\sum_{j} n_{j}^{\mathrm{ex}} V_{j}^{0}=\frac{X}{1+X}
$$

where

$$
X=\frac{\sum_{i} n_{i}^{\mathrm{ex}} V_{i}^{0}}{1-\sum_{i} n_{i}^{\mathrm{ex}} V_{i}^{0}}
$$

Here $X$ is the ratio of the occupied volume to the available volume. Finally, $n_{i}^{\mathrm{ex}}$ can be written as

$$
n_{i}^{\mathrm{ex}}=\frac{(1-R)}{V_{i}^{0}} \frac{Q_{i}}{\lambda_{i} \exp \left(-1 / I_{i} V_{i}^{0} \lambda_{i}\right)-Q_{i}} .
$$

The solution obtained in this model is very simple and easy. There is no arbitrary parameter in this formalism, so it can be regarded as a unique solution. However, this theory still depends crucially on the assumption that the number density of $i$ th species is a function of the $\lambda_{i}$ alone, and it is independent of the fugacities of other kinds of baryons. As the interactions between different species become significant in hot and dense HG, this assumption is no longer valid. Moreover, one serious problem crops up, since we cannot do calculation in this model for $T>185 \mathrm{MeV}$ (and $\mu_{B}>$ $450 \mathrm{MeV})$. This particular limiting value of temperature and baryon chemical potential depends significantly on the masses and the degeneracy factors of the baryonic resonances considered in the calculation.

In order to remove above discrepancies, Mishra and Singh $[32,33]$ have proposed a thermodynamically consistent EOS for a hot and dense HG using Boltzmann's statistics. They have proposed an alternative way to solve the transcendental equation (22). We have extended this model by using quantum statistics into the grand canonical partition function; so that our model works even for the extreme values of temperature and baryon chemical potential. Thus (20) can be rewritten as follows [31]:

$$
I_{i}=\frac{g_{i}}{6 \pi^{2} T} \int_{0}^{\infty} \frac{k^{4} d k}{\sqrt{k^{2}+m_{i}^{2}}} \frac{1}{\left[\exp \left(E_{i} / T\right)+\lambda_{i}\right]},
$$

and (22) takes the following form after using the quantum statistics in the partition function:

$$
n_{i}^{\mathrm{ex}}=(1-R) I_{i} \lambda_{i}-I_{i} \lambda_{i}^{2} \frac{\partial R}{\partial \lambda_{i}}+\lambda_{i}^{2}(1-R) I_{i}^{\prime},
$$

where $I_{i}^{\prime}$ is the partial derivative of $I_{i}$ with respect to $\lambda_{i}$. We can write $R$ in an operator equation form as follows [32, 33, 90, 91]:

$$
R=R_{1}+\widehat{\Omega} R,
$$

where $R_{1}=R^{0} /\left(1+R^{0}\right)$ with $R^{0}=\sum n_{i}^{0} V_{i}^{0}+\sum I_{i}^{\prime} V_{i}^{0} \lambda_{i}^{2} ; n_{i}^{0}$ is the density of pointlike baryons of $i$ th species and the operator $\widehat{\Omega}$ has the following form:

$$
\widehat{\Omega}=-\frac{1}{1+R^{0}} \sum_{i} n_{i}^{0} V_{i}^{0} \lambda_{i} \frac{\partial}{\partial \lambda_{i}} .
$$

Using the Neumann iteration method and retaining the series upto $\widehat{\Omega}^{2}$ term, we get

$$
R=R_{1}+\widehat{\Omega} R_{1}+\widehat{\Omega}^{2} R_{1} .
$$

After solving (39), we finally get the expression for total pressure [70] of the hadron gas as follows:

$$
P^{\mathrm{ex}}=T(1-R) \sum_{i} I_{i} \lambda_{i}+\sum_{j} P_{j}^{\mathrm{meson}} .
$$

Here $P_{j}^{\text {meson }}$ is the pressure due to $j$ th type of meson.

Here we emphasize that we consider the repulsion arising only between a pair of baryons and/or antibaryons because we assign each of them exclusively a hardcore volume. In order to make the calculation simple, we have taken an equal volume $V^{0}=4 \pi r^{3} / 3$ for each type of baryons with 
a hardcore radius $r=0.8 \mathrm{fm}$. We have considered in our calculation all baryons and mesons and their resonances having masses up to a cutoff value of $2 \mathrm{GeV} / \mathrm{c}^{2}$ and lying in the HG spectrum. Here only those resonances which possess well-defined masses and widths have been incorporated in the calculations. Branching ratios for sequential decays have been suitably accounted, and in the presence of several decay channels, only dominant mode is included. We have also imposed the condition of strangeness neutrality strictly by putting $\sum_{i} S_{i}\left(n_{i}^{s}-\bar{n}_{i}^{s}\right)=0$, where $S_{i}$ is the strangeness quantum number of the $i$ th hadron and $n_{i}^{s}\left(\bar{n}_{i}^{s}\right)$ is the strange (antistrange) hadron density, respectively. Using this constraint equation, we get the value of strange chemical potential in terms of $\mu_{B}$. Having done all these things, we proceed to calculate the energy density of each baryon species $i$ by using the following formula:

$$
\epsilon_{i}^{\mathrm{ex}}=\frac{T^{2}}{V} \frac{\partial \ln Z_{i}^{\mathrm{ex}}}{\partial T}+\mu_{i} n_{i}^{\mathrm{ex}} .
$$

Similarly, entropy density is

$$
s=\frac{\epsilon_{i}^{\mathrm{ex}}+P^{\mathrm{ex}}-\mu_{B} n_{B}-\mu_{S} n_{S}}{T} .
$$

It is evident that this approach is more simple in comparison to other thermodynamically consistent excluded-volume approaches which often possess transcendental final expressions $[69,86]$. Our approach does not involve any arbitrary parameter in the calculation. Moreover, this approach can be used for extremely low as well as extremely large values of $T$ and $\mu_{B}$, where all other approaches fail to give a satisfying result since we do not use Boltzmann's approximation in our calculation.

\section{Statistical and Thermodynamical Consistency}

Recently, question of statistical and thermodynamical consistency in excluded-volume models for HG has widely been discussed [77]. In this section, we reexamine the issue of thermodynamical and statistical consistency of the excludedvolume models. In RGSG model [69], the single particle grand canonical partition function (9) can be rewritten as follows:

$$
\begin{aligned}
Z_{G}^{\mathrm{ex}}(V, T, \mu)=\sum_{N=0}^{\infty} & \exp \left(\frac{\mu N}{T}\right) \frac{\left(V-V^{0} N\right)^{N}}{N !} \\
& \times \theta\left(V-V^{0} N\right) z^{N},
\end{aligned}
$$

where,

$$
z(T)=\frac{g}{2 \pi^{2}} \int_{0}^{\infty} k^{2} d k \exp \left[-\frac{\left(k^{2}+m^{2}\right)^{1 / 2}}{T}\right] .
$$

Here, in this model, $N$ in the available volume $\left(V-V^{0} N\right)$ is independent of $\mu$. Differentiating (43) with respect to $\mu$, we get the following equation:

$$
\frac{\partial Z_{G}^{\mathrm{ex}}}{\partial \mu}=\sum_{N=0}^{\infty} \frac{N}{T} \exp \left(\frac{\mu N}{T}\right) \frac{\left(V-V^{0} N\right)^{N}}{N !} \theta\left(V-V^{0} N\right) z^{N} .
$$

Multiplying both sides of (45) by $T / Z_{G}^{\text {ex }}$, we get

$$
\begin{aligned}
\frac{T}{Z_{G}^{\mathrm{ex}}} \frac{\partial Z_{G}^{\mathrm{ex}}}{\partial \mu}=\frac{1}{Z^{\mathrm{ex}}} \sum_{N=0}^{\infty} & N \exp \left(\frac{\mu N}{T}\right) \frac{\left(V-V^{0} N\right)^{N}}{N !} \\
& \times \theta\left(V-V^{0} N\right) z^{N} .
\end{aligned}
$$

We know that the expressions for statistical and thermodynamical averages of number of baryons are as follows:

$$
\begin{gathered}
\langle N\rangle=\frac{1}{Z_{G}^{\mathrm{ex}}} \sum_{N=0}^{\infty} N \exp \left(\frac{\mu N}{T}\right) \frac{\left(V-V^{0} \bar{N}\right)^{N}}{N !} z^{N}, \\
\bar{N}=T \frac{\partial \ln Z_{G}^{\mathrm{ex}}}{\partial \mu}=\frac{T}{Z_{G}^{\mathrm{ex}}} \frac{\partial Z_{G}^{\mathrm{ex}}}{\partial \mu},
\end{gathered}
$$

respectively. Using (47) in (46), we get [77]

$$
\bar{N}=\langle N\rangle \text {. }
$$

Thus, we see that in RGSG model, thermodynamical average of number of baryons is exactly equal to the statistical average of number of baryons. Similarly in this model, we can show that

$$
\bar{E}=\langle E\rangle .
$$

Now, we calculate the statistical and thermodynamical averages of number of baryons in our excluded-volume model. The grand canonical partition function in our model (i.e., (18)) can take the following form:

$$
Z^{\text {ex }}(V, T, \mu)=\sum_{N=0}^{\infty} \exp \left(\frac{\mu N}{T}\right) \frac{\left(V-V^{0} \bar{N}\right)^{N}}{N !} z^{N},
$$

where $z$ is given by (44). We use Boltzmann's statistics for the sake of convenience and consider only one species of baryons. In our model, $\bar{N}$, present in the available volume $\left(V-V^{0} \bar{N}\right)$, is $\mu$ dependent. However, for multicomponent system, one cannot use "fixed $N$ ", because in this case van-der Waals approximation is not uniquely defined $[92,93]$. So, we use average $N$ in our multicomponent grand partition function. However, at high temperatures it is not possible to use one component van-der Waals description for a system of various species with different masses $[92,93]$. Now differentiating (50) with respect to $\mu$, we get

$$
\begin{aligned}
\frac{\partial Z^{\mathrm{ex}}}{\partial \mu}= & \sum_{N=0}^{\infty} \frac{N}{T} \exp \left(\frac{\mu N}{T}\right) \frac{\left(V-V^{0} \bar{N}\right)^{N}}{N !} z^{N} \\
& -\sum_{N=0}^{\infty} \exp \left(\frac{\mu N}{T}\right) \frac{\left(V-V^{0} \bar{N}\right)^{N-1}}{N-1 !} z^{N} V^{0} \frac{\partial \bar{N}}{\partial \mu} .
\end{aligned}
$$


Multiplying both sides of (51) by $T / Z^{\text {ex }}$, we get

$$
\begin{aligned}
\frac{T}{Z^{\mathrm{ex}}} \frac{\partial Z^{\mathrm{ex}}}{\partial \mu}= & \frac{1}{Z^{\mathrm{ex}}} \sum_{N=0}^{\infty} N \exp \left(\frac{\mu N}{T}\right) \frac{\left(V-V^{0} \bar{N}\right)^{N}}{N !} z^{N} \\
& -\frac{T}{Z^{\mathrm{ex}}} \sum_{N=0}^{\infty} \exp \left(\frac{\mu N}{T}\right) \frac{\left(V-V^{0} \bar{N}\right)^{N-1}}{N-1 !} \\
& \times z^{N} V^{0} \frac{\partial \bar{N}}{\partial \mu} .
\end{aligned}
$$

Using the definitions (47), (52) can take the following form:

$$
\bar{N}=\langle N\rangle-\frac{T}{Z^{\mathrm{ex}}} \sum_{N=0}^{\infty} \exp \left(\frac{\mu N}{T}\right) \frac{\left(V-V^{0} \bar{N}\right)^{N-1}}{N-1 !} z^{N} V^{0} \frac{\partial \bar{N}}{\partial \mu},
$$

or

$$
\bar{n}=\langle n\rangle-T\left\langle\frac{n^{0}}{(1-R)} V^{0} \frac{\partial \bar{n}}{\partial \mu}\right\rangle
$$

Here $\bar{n}$ is the thermal average of number density of baryons, $n^{0}$ is the number density of pointlike baryons, and

$$
\begin{aligned}
& \left\langle\frac{n^{0}}{(1-R)} V^{0} \frac{\partial \bar{n}}{\partial \mu}\right\rangle \\
& \quad=\frac{1}{Z^{e x}} \sum_{N=0}^{\infty} \exp \left(\frac{\mu N}{T}\right) \frac{\left(V-V^{0} \bar{N}\right)^{N}}{N !} z^{N} \\
& \quad \times\left(\frac{N}{\left(V-V^{0} \bar{N}\right)} V^{0} \frac{\partial \bar{N}}{\partial \mu}\right) .
\end{aligned}
$$

The second term in (54) is the redundant one and arises because $\bar{N}$, present in the available volume $\left(V-V^{0} \bar{N}\right)$, is a function of $\mu$. We call this term "correction term". In Figure 2, we have shown the variation of thermodynamical average of the number density of baryons and the "correction term" with respect to $T$ at $\mu_{B}=400 \mathrm{MeV}$. We see that there is an almost negligible contribution of this "correction term" to thermodynamical average of number density of baryons. Although, due to this "correction term", the statistical average of the number density of baryons is not exactly equal to its thermodynamical average, the difference is so small that it can be neglected. Similarly, we can show that such redundant terms appear while calculating statistical average of energy density of baryons and arise due to the temperature dependence of $\bar{N}$. Such terms again give negligible contribution to thermodynamical average of the energy density of baryons. Here, we see that the statistical and thermodynamical averages of physical quantities such as number density and energy density are approximately equal to each other in our model also. Thus, our excluded-volume model is not exactly thermodynamically consistent, but it can safely be taken as consistent because the correction term in the averaging procedure appears as negligibly small.

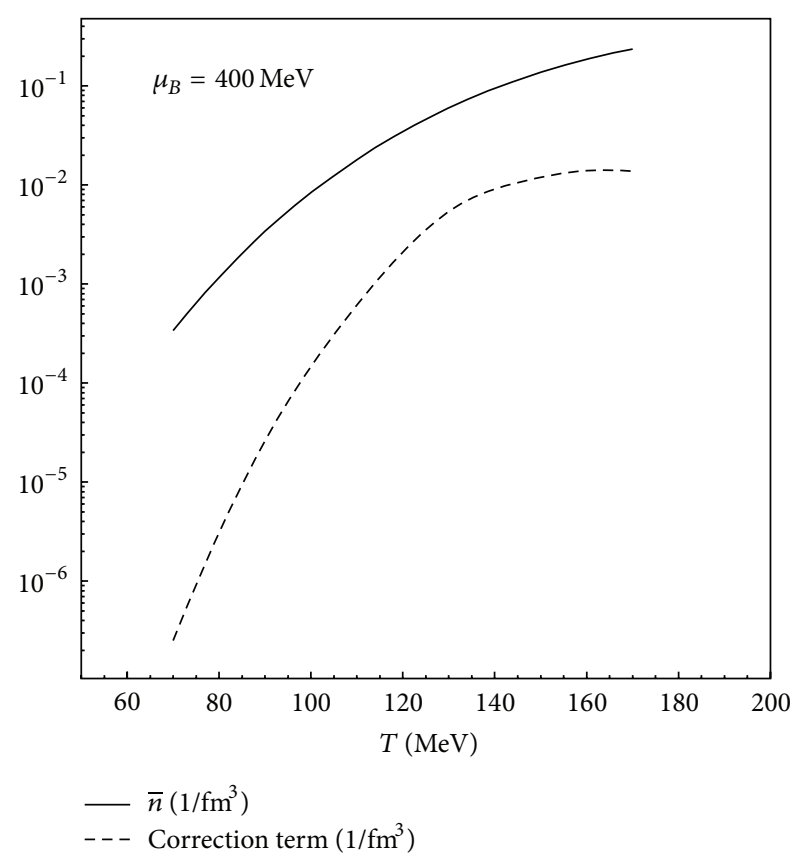

FIGURE 2: Variation of thermodynamical average of the number density of baryons and the "correction term" with respect to $T$ at constant $\mu_{B}$.

\section{Comparisons between Model Results and Experimental Data}

In this section, we review various features of hadron gas and present our comparisons between the results of various HG models and the experimental data.

4.1. Chemical Freeze-Out Criteria. Thermal models provide a systematic study of many important properties of hot and dense HG at chemical freezeout (where inelastic interactions cease). To establish a relation between chemical freezeout parameters $\left(T, \mu_{B}\right)$ and $\sqrt{s_{N N}}$, a common method is used to fit the experimental hadron ratios. Many papers [30, 49, 50, 94-96] have appeared in which $T$ and $\mu_{B}$ are extracted in terms of $\sqrt{s_{N N}}$. In [30], various hadron ratios are analyzed from $\sqrt{s_{N N}}=2.7 \mathrm{GeV}$ to $200 \mathrm{GeV}$, and chemical freeze-out parameters are parameterized in terms of $\sqrt{s_{N N}}$ by using following expressions:

$$
\begin{gathered}
T(\mathrm{MeV})=T_{\lim }\left(1-\frac{1}{a+\left(\exp \left(\sqrt{s_{N N}}(\mathrm{GeV})\right)-b\right) / c}\right), \\
\mu_{B}=\frac{d}{1+e \sqrt{s_{N N}}} .
\end{gathered}
$$

Here, $a, b, c, d, e$, and $T_{\lim }$ (the limiting temperature) are fitting parameters. Various authors $[94,95]$ have included the strangeness suppression factor $\left(\gamma_{s}\right)$ in their model while extracting the chemical freeze-out parameters. In the thermal model, $\gamma_{s}$ is used to account for the partial equilibration of the strange particles. Such situation may arise in the elementary 

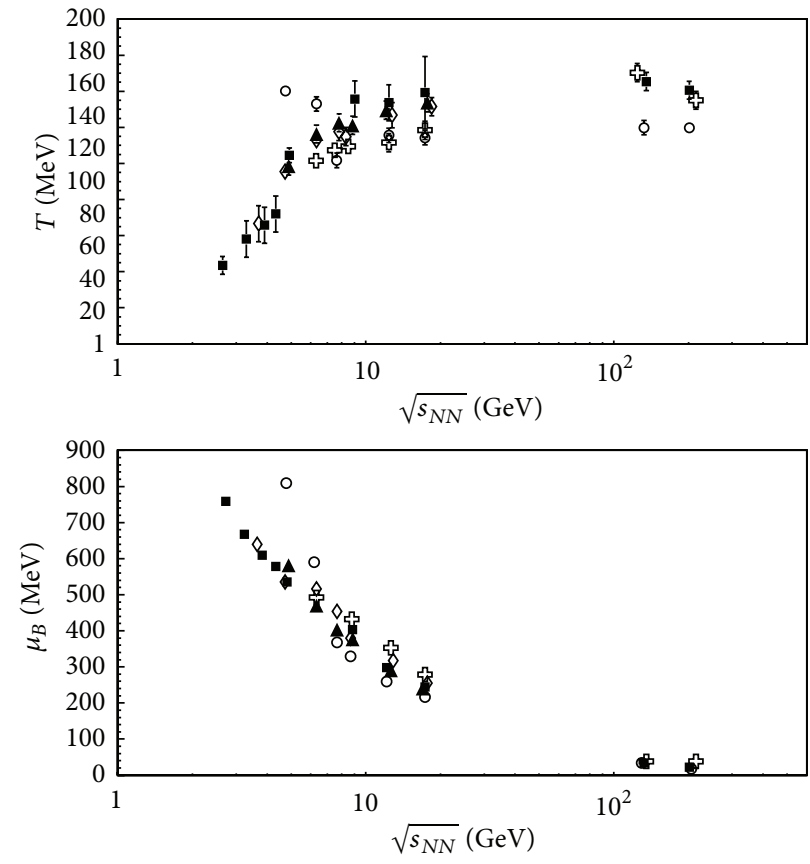

- Andronic et al. $(d N / d y) \quad$ ○ Letessier et al. $(4 \pi)$
$\diamond$ Andronic et al. $(4 \pi)$
$\begin{aligned} & \text { \& Becattini et al. }(4 \pi) \\ & \text { D Dumitru et al. }(4 \pi)\end{aligned}$

FIGURE 3: The energy dependence of chemical freeze-out temperature and baryon chemical potential in various studies. Figure is taken from [30].

p-p collisions and/or peripheral A-A collisions, and mostly the use of $\gamma_{s}$ is warranted in such cases [94, 97]. Moreover, $\gamma_{s} \approx 1$ has been found in the central collisions at RHIC $[98,99]$. We do not require $\gamma_{s}$ in our model as an additional fitting parameter because we assume that strangeness is also fully equilibrated in the HG. Also, it has been pointed out that inclusion of $\gamma_{s}$ in thermal model analysis does not affect the values of fitting parameters $T$ and $\mu_{B}$ much [30]. Dumitru et al. [96] have used inhomogeneous freeze-out scenario for the extraction of $T$ and $\mu_{B}$ at various $\sqrt{s_{N N}}$. In a recent paper [100], condition of vanishing value of $\kappa \sigma^{2}$ or equivalently $m_{4}=3 \chi^{2}$ is used to describe the chemical freezeout line where $\kappa, \sigma, m_{4}$, and $\chi$ are kurtosis, the standard deviation, fourth order moment, and susceptibility, respectively. In [101], first time experimental data on $\kappa \sigma^{2}$ and $S \sigma$, here $S$ is skewness, has been compared with the lattice QCD calculations and hadron resonance gas model to determine the critical temperature $\left(T_{c}\right)$ for the QCD phase transition. Recently, it is shown that the freeze-out parameters in heavy-ion collisions can be determined by comparing the lattice QCD results for the first three cumulants of net electric charge fluctuations with the experimental data [102]. In Figure 3, we have shown the energy dependence of thermal parameters $T$ and $\mu_{B}$ extracted by various authors. In all the studies, similar behaviour is found except in the Letessier and Rafelski [95], which may be due to usage of many additional free parameters such as light quark occupancy factor $\left(\gamma_{q}\right)$ and an isospin fugacity. We have also extracted freeze-out parameters by fitting the experimental particleratios from the lowest SIS energy to the highest RHIC energy using our model [31]. For comparison, we have shown the values obtained in other models, for example, IHG model, Cleymans-Suhonen model, and RGSG model, in Table 1. We then parameterize the variables $T$ and $\mu_{B}$ in terms of $\sqrt{s_{N N}}$ as follows [103]:

$$
\begin{gathered}
\mu_{B}=\frac{a}{1+b \sqrt{s_{N N}}}, \\
T=c-d \mu_{B}^{2}-e \mu_{B}^{4},
\end{gathered}
$$

where the parameters $a, b, c, d$, and $e$ have been determined from the best fits: $a=1.482 \pm 0.0037 \mathrm{GeV}, b=0.3517 \pm$ $0.009 \mathrm{GeV}^{-1}, c=0.163 \pm 0.0021 \mathrm{GeV}, d=0.170 \pm$ $0.02 \mathrm{GeV}^{-1}$, and $e=0.015 \pm 0.01 \mathrm{GeV}^{-3}$. The systematic error of the fits can be estimated via quadratic deviation $\delta^{2}$ [30] defined as follows:

$$
\delta^{2}=\sum_{i} \frac{\left(R_{i}^{\text {exp }}-R_{i}^{\text {therm }}\right)^{2}}{\left(R_{i}^{\text {therm }}\right)^{2}}
$$

where $R_{i}^{\exp }$ and $R_{i}^{\text {therm }}$ are the experimental data and thermal model result of either the hadron yield or the ratio of hadron yields, respectively.

In this analysis, we have used full phase space $(4 \pi)$ data at all center-of-mass energies except at RHIC energies where only midrapidity data are available for all the ratios. Moreover, the midrapidity and full phase space data at these 
TABLE 1: Thermal parameters $\left(T, \mu_{B}\right)$ values obtained by fitting the experimental particle ratios in different model calculations.

\begin{tabular}{lccccccccccccc}
\hline \multirow{2}{*}{$\sqrt{s_{N N}}(\mathrm{GeV})$} & \multicolumn{3}{c}{ IHG model } & \multicolumn{3}{c}{ RGSG model } & \multicolumn{3}{c}{ Cleymans-Suhonen model } & \multicolumn{3}{c}{ Our model } \\
& $T$ & $\mu_{B}$ & $\delta^{2}$ & $T$ & $\mu_{B}$ & $\delta^{2}$ & $T$ & $\mu_{B}$ & $\delta^{2}$ & $T$ & $\mu_{B}$ & $\delta^{2}$ \\
\hline 2.70 & 60 & 740 & 0.85 & 60 & 740 & 0.75 & 70 & 753 & 1.19 & 70 & 760 & 1.15 \\
3.32 & 80 & 670 & 0.89 & 78 & 680 & 0.34 & 89 & 686 & 0.75 & 90 & 670 & 0.45 \\
3.84 & 100 & 645 & 0.50 & 86 & 640 & 0.90 & 101 & 639 & 0.37 & 100 & 640 & 0.34 \\
4.32 & 101 & 590 & 0.70 & 100 & 590 & 0.98 & 109 & 600 & 0.17 & 105 & 600 & 0.23 \\
8.76 & 140 & 380 & 0.45 & 145 & 406 & 0.62 & 144 & 386 & 0.05 & 140 & 360 & 0.25 \\
12.3 & 148 & 300 & 0.31 & 150 & 298 & 0.71 & 153 & 300 & 0.03 & 150 & 276 & 0.20 \\
17.3 & 160 & 255 & 0.25 & 160 & 240 & 0.62 & 158.6 & 228 & 0.63 & 155 & 206 & 0.27 \\
130 & 172.3 & 35.53 & 0.10 & 165.5 & 38 & 0.54 & 165.8 & 35.84 & 0.15 & 163.5 & 32 & 0.05 \\
200 & 172.3 & 23.53 & 0.065 & 165.5 & 25 & 0.60 & 165.9 & 23.5 & 0.10 & 164 & 20 & 0.05 \\
\hline
\end{tabular}

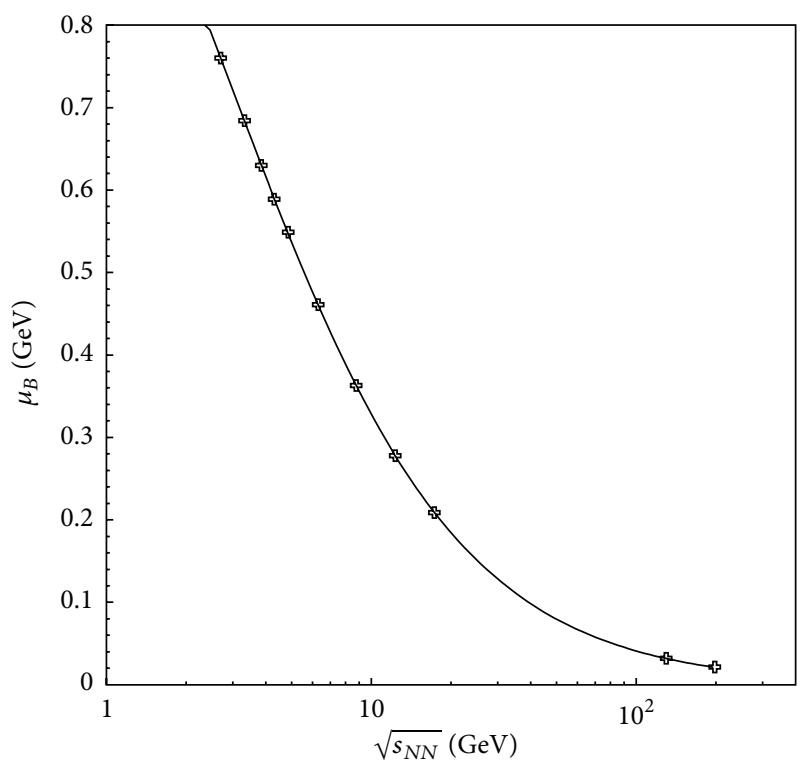

FIGURE 4: Variation of baryon chemical potential with respect to centre-of-mass energy in our model.

energies differ only slightly as pointed out by Alt and NA49 Collaboration for $K^{+} / \pi^{+}$and $K^{-} / \pi^{-}$ratios [104]. In Figure 4, we have shown the parametrization of the freeze-out values of baryon chemical potential with respect to $\sqrt{s_{N N}}$, and similarly in Figure 5, we have shown the chemical freeze-out curve between temperature and baryon chemical potential [31].

4.2. Hadron Ratios. In an experimental measurement of various particle ratios at various centre-of-mass energies [105-111], it is found that there is an unusual sharp variation in the $\Lambda / \pi^{-}$ratio increasing up to the peak value. This strong variation of the ratio with energy indicates the critical temperature of QCD phase transition [112] between HG and QGP $[113,114]$, and a nontrivial information about the critical temperature $T_{C} \approx 176 \mathrm{MeV}$ has been extracted [114]. Figure 6 shows the variation of $\Lambda / \pi^{-}$with $\sqrt{s_{N N}}$. We compare the experimental data with various thermal models [31, 63, 69]

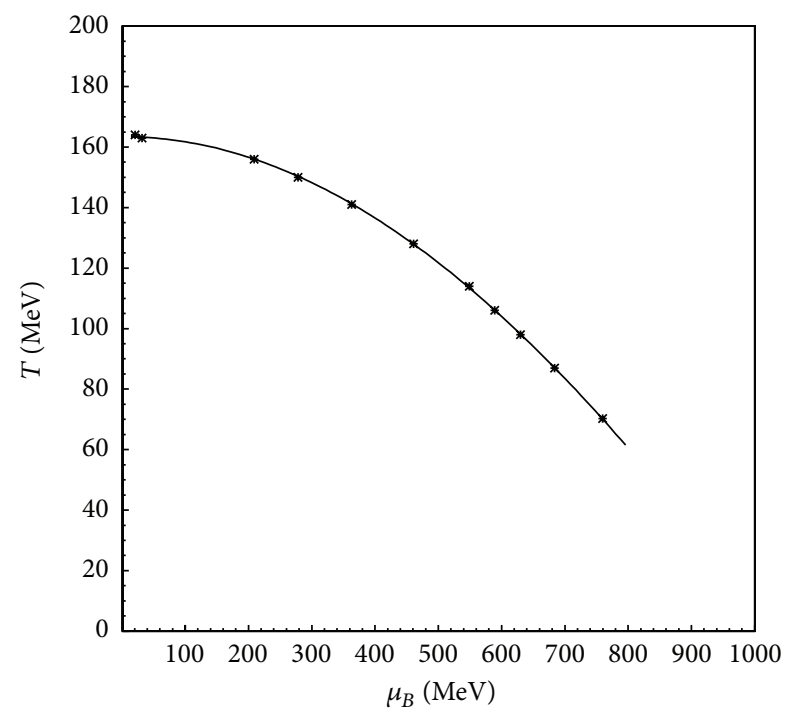

FIGURE 5: Variation of chemical freeze-out temperature with respect to baryon chemical potential in our model.

and find that our model calculation gives much better fit to the experimental data in comparison to other models. We get a sharp peak around centre-of-mass energy of $5 \mathrm{GeV}$, and our results thus almost reproduce all the features of the experimental data.

In Figure 7, we have shown the variations of $\phi / \pi$ ratio with $\sqrt{s_{N N}}$. The $\phi$ yields in the thermal models are often much higher in comparison to data. We notice that no thermal model can suitably account for the multiplicity ratio of multistrange particle since $\phi$ is $s \bar{s}$ hidden-strange quark combination. However, quark coalescence model assuming a QGP formation has been claimed to explain the results $[115,116]$ successfully. In the thermal models, the results for the multistrange particles raise doubt over the degree of chemical equilibration for strangeness reached in the $\mathrm{HG}$ fireball. We can use an arbitrary parameter $\gamma_{s}$ as used in several models. The failures of thermal models in these cases may indicate the presence of QGP formation, but it is still not clear. In Figure 8, we have shown the energy dependence of antiparticle to particle ratios; for example, $K^{-} / K^{+}, \bar{p} / p$, 


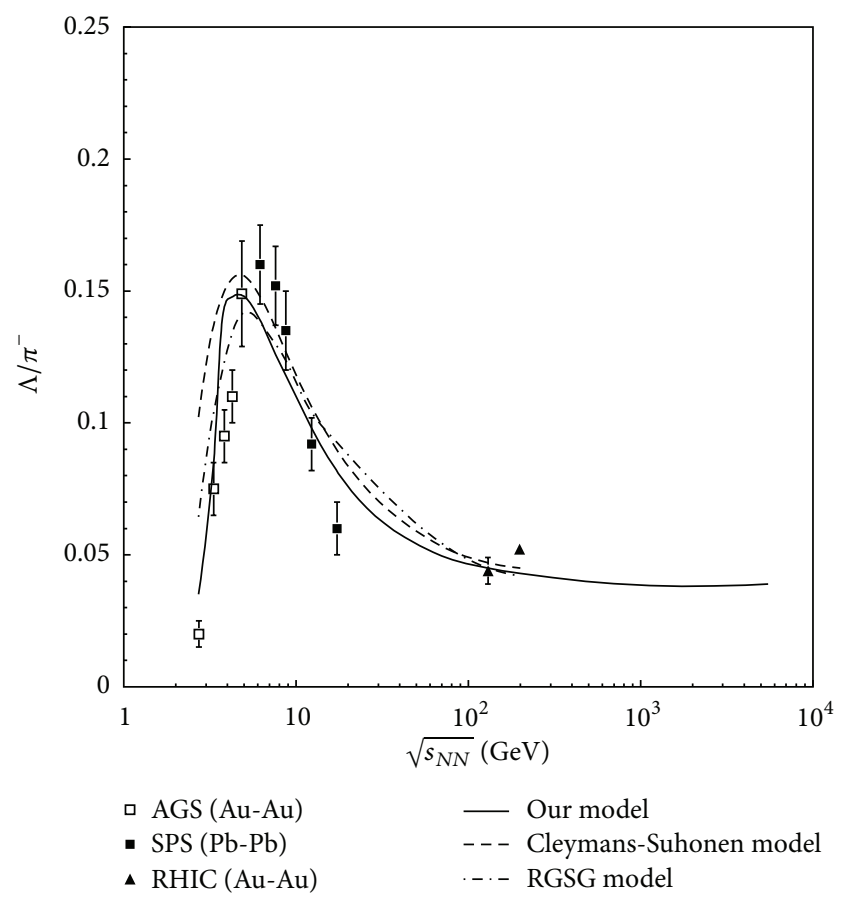

Figure 6: The energy dependence of the $\Lambda / \pi^{-}$ratio. Lines are the results of various thermal models $[31,63,69]$. Points are the experimental data $[104,117-121]$. RHIC data are at midrapidity.

$\bar{\Lambda} / \Lambda$, and $\bar{\Xi}^{+} / \Xi^{-}$. These ratios increase sharply with respect to $\sqrt{s_{N N}}$ and then almost saturate at higher energies reaching the value equal to 1.0 at LHC energy. On comparison with the experimental data we find that almost all the thermal models describe these data successfully at all the center-ofmass energies. However, RGSG model [69] fails to describe the data at SPS and RHIC energies in comparison to other models [31].

4.3. Thermodynamical Properties. We present the thermal model calculations of various thermodynamical properties of HG such as entropy per baryon $\left(s / n_{B}\right)$ and energy density and compare the results with the predictions of a microscopic model URASiMA event generator developed by Sasaki [122]. URASiMA (ultrarelativistic AA collision simulator based on multiple scattering algorithm) is a microscopic model which includes the realistic interactions between hadrons. In URASiMA event generator, molecular-dynamical simulations for a system of a HG are performed. URASiMA includes the multibody absorptions, which are the reverse processes of multiparticle production and are not included in any other model. Although, URASiMA gives a realistic EOS for hot and dense HG, it does not include antibaryons and strange particles in their simulation, which is very crucial. In Figure 9 , we have plotted the variation of $s / n_{B}$ with respect to temperature $(T)$ at fixed net baryon density $\left(n_{B}\right) . s / n_{B}$ calculated in our model shows a good agreement with the results of Sasaki [122] in comparison to other excludedvolume models. It is found that thermal model approach, which incorporates macroscopic geometrical features gives

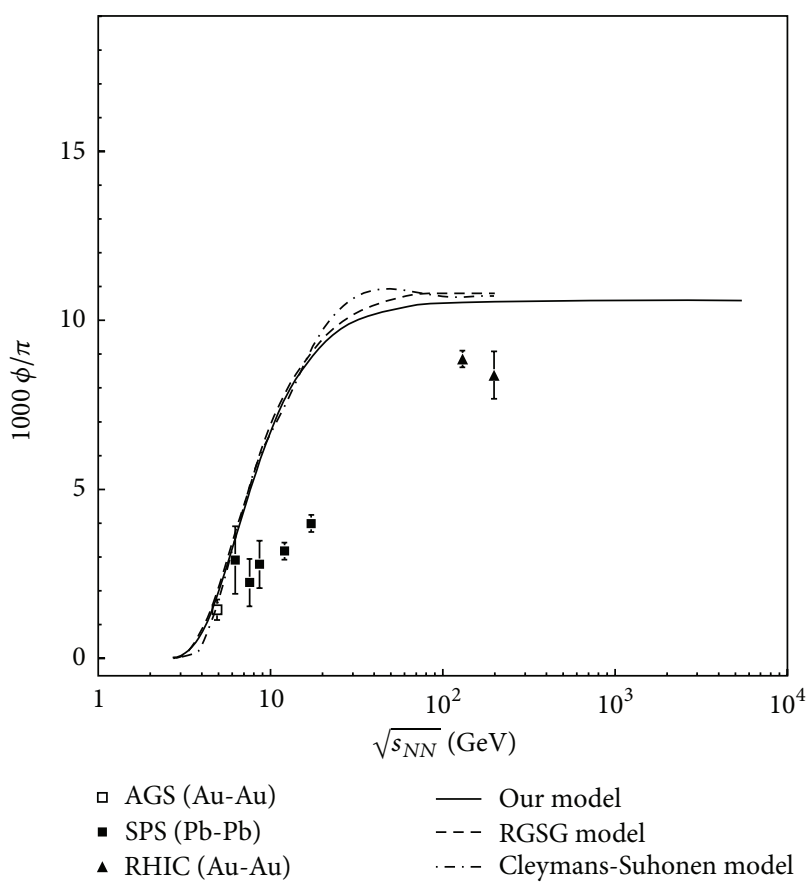

Figure 7: The energy dependence of $\phi / \pi$ ratio. Lines are the results of various thermal models $[31,63,69]$. Points are the experimental data $[104,117-121]$. RHIC data are at midrapidity.

a close results with the simulation involving microscopic interactions between hadrons. There are various parameters such as coupling constants of hadrons appear in URASiMA model due to interactions between hadrons. It is certainly encouraging to find an excellent agreement between the results obtained with two widely different approaches.

Figure 10 represents the variation of the energy density of $\mathrm{HG}$ with respect to $T$ at constant $n_{B}$. Again our model calculation is more closer to the result of URASiMA in comparison to other excluded-volume models. Energy density increases very slowly with the temperature initially and then rapidly increases at higher temperatures.

4.4. Causality. One of the deficiencies of excluded-volume models is the violation of causality in the hot and dense hadron gas; that is, the sound velocity $c_{s}$ is larger than the velocity of light $c$ in the medium. In other words, $c_{s}>1$, in the unit of $c=1$, means that the medium transmits information at a speed faster than $c$ [74]. Since, in this paper we are discussing the results of various excluded-volume models, it would be interesting to see whether these models respect causality or not. In Figure 11, we have plotted the variations of the total hadronic pressure $P$ as a function of the energy density $\epsilon$ of the HG at a fixed entropy per particle using our model calculation [31]. We find for a fixed $s / n$ that the pressure varies linearly with respect to energy density. In Figure 12, we have shown the variation of $c_{s}\left(c_{s}^{2}=\partial P / \partial \epsilon\right.$ at fixed $s / n$ ) with respect to $s / n$. We find that $c_{s} \leq 0.58$ in our model with interacting particles. We get $c_{s}=0.58$ (i.e., $1 / \sqrt{3}$ ) for an ideal gas consisting of ultrarelativistic particles. This 

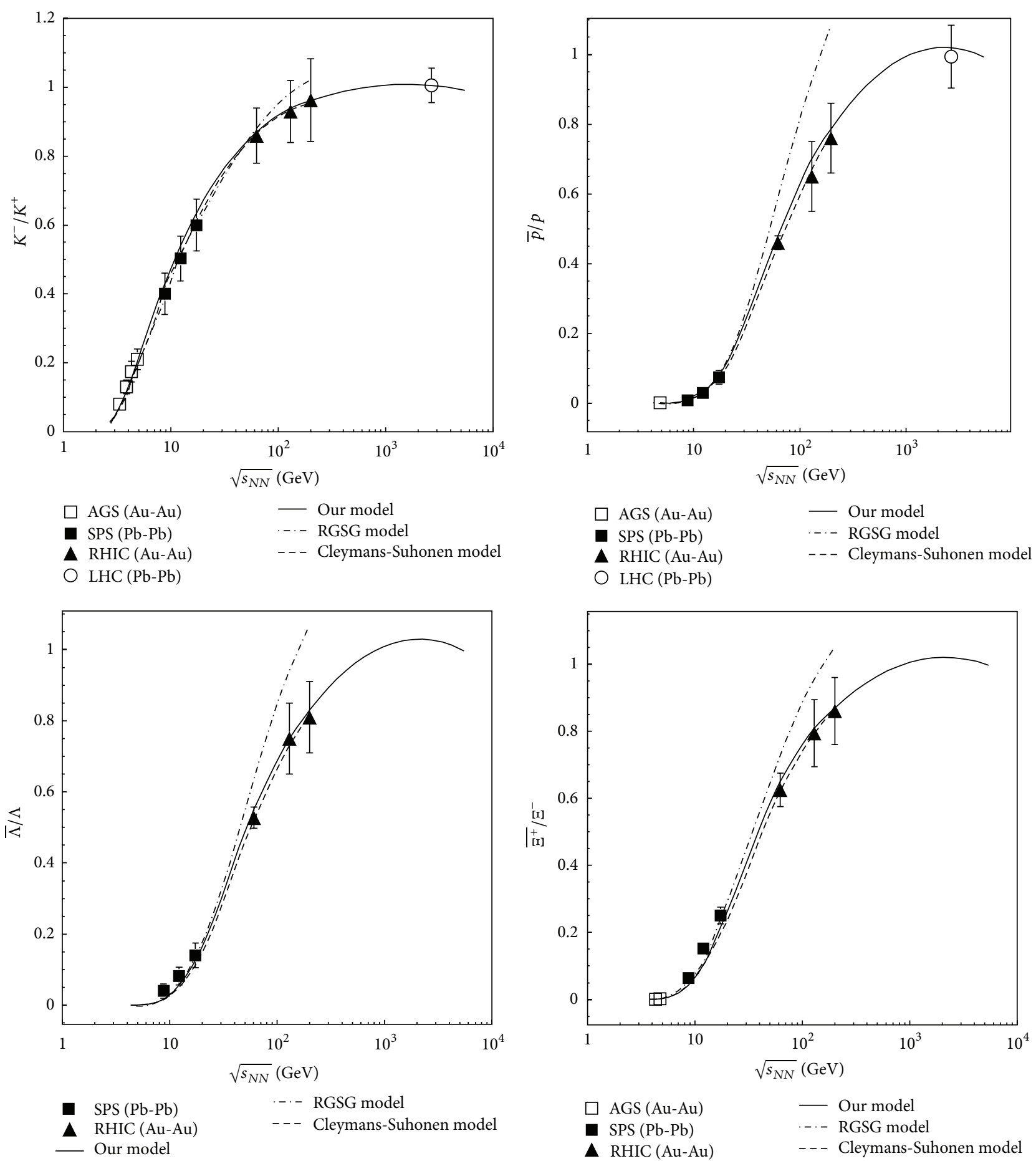

FIgure 8: The energy dependence of antihadron to hadron ratios. Points are the experimental data [104, 117-121], and lines are results of various models. RHIC and LHC data are at midrapidity.

feature endorses our viewpoint that our model is not only thermodynamically consistent but it does not also involve any violation of causality even at large density. Similarly in RGSG model [69], we do not notice that the value of $c_{s}$ exceeds 1 as shown in Figure 12. It should be mentioned that we are using full quantum statistics in all the models taken for comparisons here. However, we find that the values in the RGSG model cannot be extracted when temperature of the $\mathrm{HG}$ exceeds $250 \mathrm{MeV}$. No such restriction applies for our model.

4.5. Universal Freeze-Out Criteria. One of the most remarkable successes of thermal models is in explaining the multiplicities and the particle ratios of various particles produced 


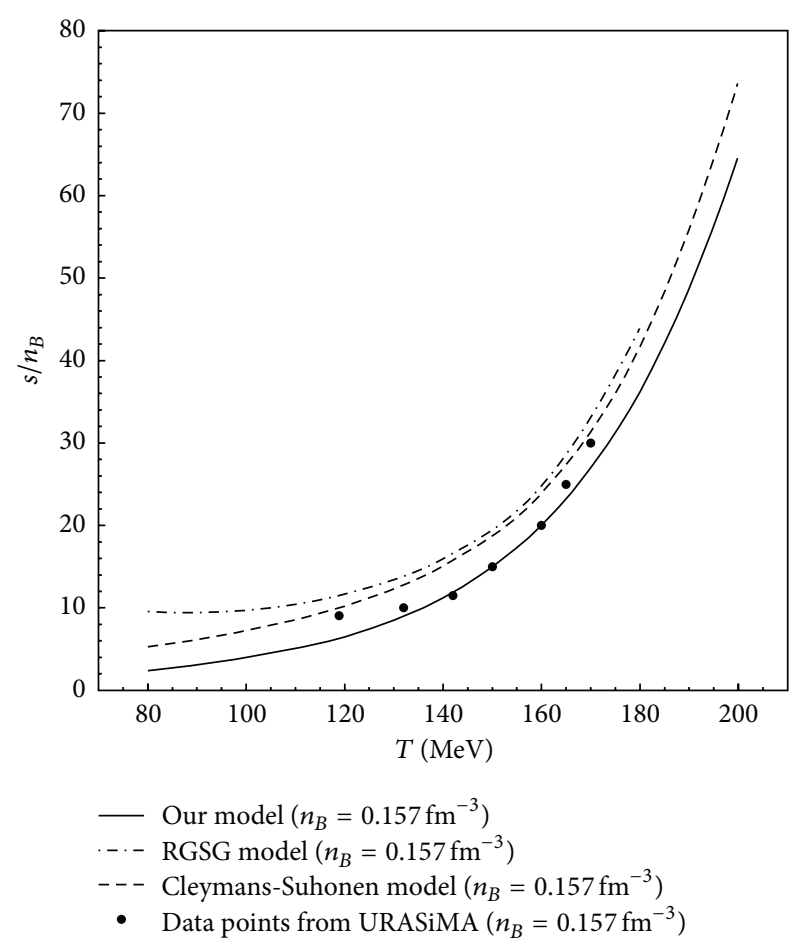

FIGURE 9: Variation of $s / n_{B}$ with respect to temperature at constant net baryon density. Lines show the results of various thermal models, and points are the data calculated by Sasaki using URASiMA event generator.

in heavy-ion experiments from the lowest SIS energy to maximum LHC energy. Some properties of thermal fireball are found to be common to all collision energies which give a universal freeze-out conditions in heavy-ion collisions. Now, we review the applicability of thermal models in deriving some useful chemical freeze-out criteria for the fireball. Recent studies [39, 48, 103, 123-125] predict that the following empirical conditions are to be valid on the entire freeze-out hypersurface of the fireball: (i) energy per hadron always has a fixed value at $1.08 \mathrm{GeV}$; (ii) sum of baryon and anti-baryon densities is $n_{B}+n_{\bar{B}}=0.12 / \mathrm{fm}^{3}$; (iii) normalized entropy density is $s / T^{3} \approx 7$. Further, Cleymans et al. [103] have found that all the above conditions separately give a satisfactory description of the chemical freeze-out parameters $T$ and $\mu_{B}$ in an IHG picture only. Moreover, it was also found that these conditions are independent of collision energy and the geometry of colliding nuclei. Furthermore, Cleymans et al. [103] have hinted that incorporation of excluded-volume correction leads to wild as well as disastrous effects on these conditions. The purpose in this section is to reinvestigate the validity of these freeze-out criteria in excluded-volume models. Along with these conditions, a condition, formulated by using percolation theory, is also proposed as a chemical freeze-out condition [124]. An assumption is made that in the baryonless region the hadronic matter freezes out due to hadron resonances and vacuum percolation, while in the baryon rich region the freeze-out takes place due to baryon percolation. Thus, the condition which describes the

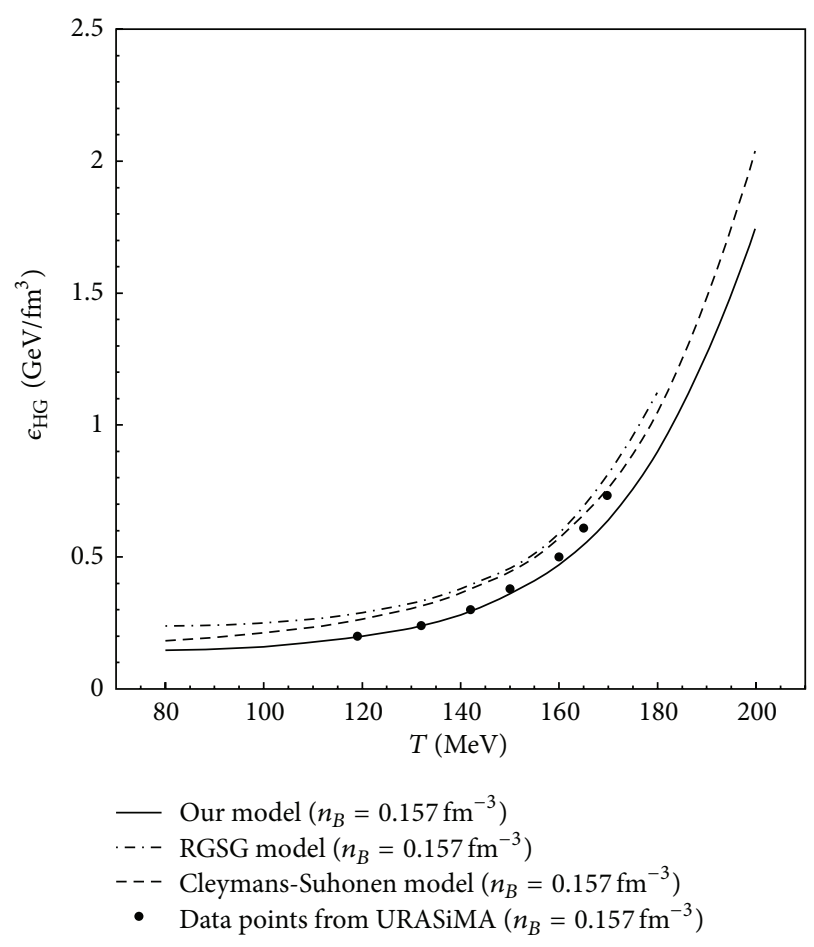

FIGURE 10: Variation of energy density with respect to temperature at constant net baryon density. Lines are the results of various thermal models and points are the data calculated by Sasaki using URASiMA event generator.

chemical freeze-out line is formulated by following equation [124]:

$$
n(T, \mu)=\frac{1.24}{V_{h}}\left[1-\frac{n_{B}(T, \mu)}{n(T, \mu)}\right]+\frac{0.34}{V_{h}} \frac{n_{B}(T, \mu)}{n(T, \mu)},
$$

where $V_{h}$ is the volume of a hadron. The numbers 1.24 and 0.34 are obtained within percolation theory [126].

In Figure 13, we have shown the variation of $E / N$ with respect to $\sqrt{s_{N N}}$ at the chemical freeze-out point of the fireball. The ratio $E / N$ shows a constant value of 1.0 in our model, and it shows also a remarkable energy independence. Similarly the curve in IHG model shows that the value for $E / N$ is slightly larger than the one as reported in [103]. However, results support the finding that $E / N$ is almost independent of energy and also of the geometry of the nuclei. Most importantly, we notice that the inclusion of the excluded-volume correction does not change the result much which is contrary to the claim of Cleymans et al. [103]. The condition $E / N \approx 1.0 \mathrm{GeV}$ was successfully used in the literature to make predictions [45] of freeze-out parameters at SPS energies of 40 and $80 \mathrm{~A} \mathrm{GeV}$ for $\mathrm{Pb}-\mathrm{Pb}$ collisions long before the data were taken [31]. Moreover, we have also shown, in Figure 13, the curves in the Cleymans-Suhonen model [63] and the RGSG model [69], and we notice a small variation with $\sqrt{s_{N N}}$ particularly at lower energies. In Figure 14, we study a possible new freeze-out criterion which was not proposed earlier. We show that the quantity entropy per particle, that is, $S / N$, yields a remarkable energy 


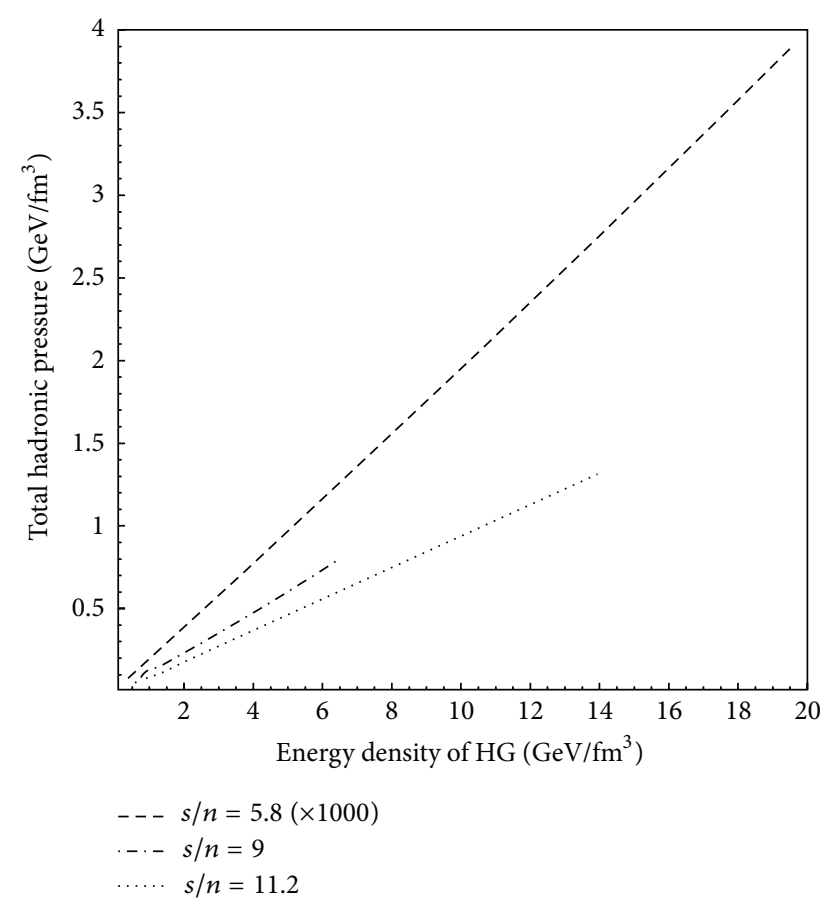

FIgURE 11: Variations of total hadronic pressure with respect to energy density of $\mathrm{HG}$ at fixed entropy per particle $s / n$. Our calculations show linear relationship, and slope of the lines gives square of the velocity of sound $c_{s}^{2}[31]$.

independence in our model calculation. The quantity $S / N \approx$ 7.0 describes the chemical freeze-out criteria and is almost independent of the centre-of-mass energy in our model calculation. However, the results below, $\sqrt{s_{N N}}=6 \mathrm{GeV}$, do not give a promising support to our criterion and reveal some energy dependence also. This criterion thus indicates that the possible use of excluded-volume models and the thermal descriptions at very low energies is not valid for the HG. Similar results were obtained in the RGSG, Cleymans-Suhonen, and IHG models also [31]. The conditions, that is, $E / N \approx$ $1.0 \mathrm{GeV}$ and $S / N \approx 7.0$, at the chemical freeze-out form a constant hypersurface from where all the particles freeze out and all kinds of inelastic collisions cease simultaneously and fly towards the detectors. Thus all particles attain thermal equilibrium at the line of chemical freeze-out, and when they come out from the fireball, they have an almost constant energy per particle $(\approx 1.0)$ and entropy per particle $(\approx 7.0)$. Moreover, these values are independent of the initial collision energy as well as the geometry of the colliding nuclei.

Our finding lends support to the crucial assumption of HG fireball achieving chemical equilibrium in the heavyion collisions from the lowest SIS to RHIC energy, and the EOS of the HG developed by us indeed gives a proper description of the hot and dense fireball and its subsequent expansion. However, we still do not get any information regarding QGP formation from these studies. The chemical equilibrium once attained by the hot and dense HG removes any memory regarding QGP existing in the fireball before HG phase. Furthermore, in a heavy-ion collision, a large amount of kinetic energy becomes available, and part of it is

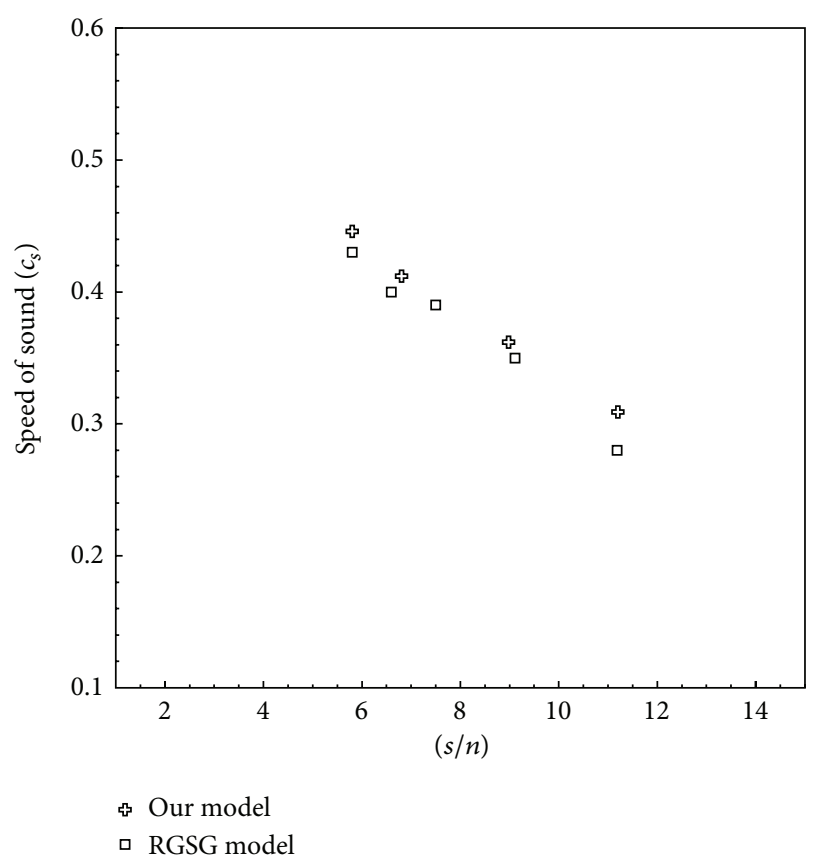

FIGURE 12: Variation of velocity of sound in the hot, dense HG medium with respect to entropy per particle $(s / n)$.

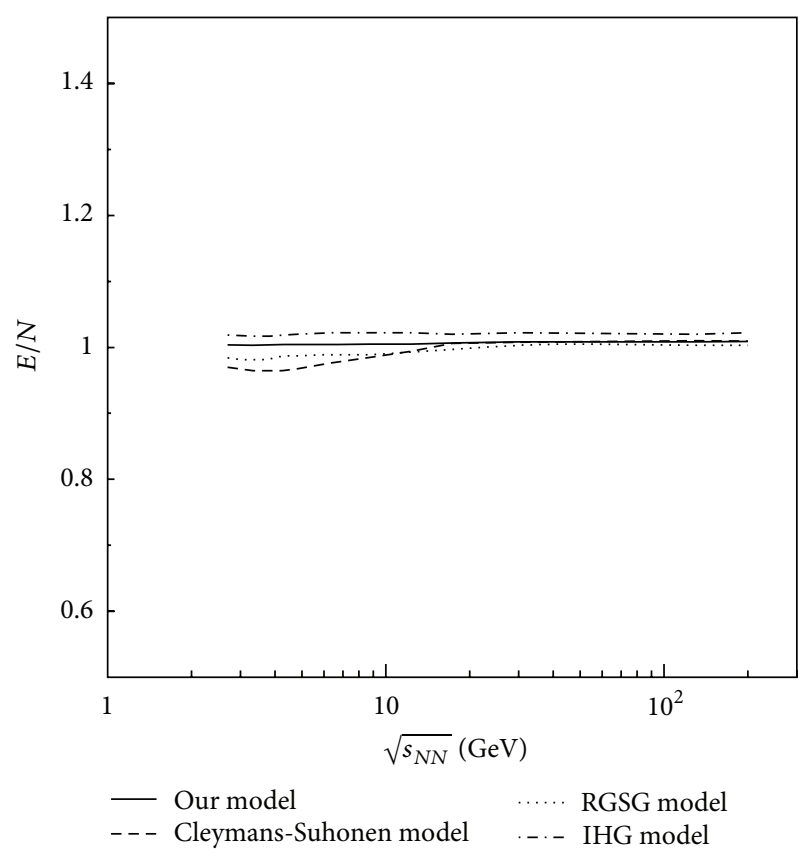

FIGURE 13: Variation of $E / N$ with $\sqrt{s_{N N}}$. IHG model calculation is shown by dash-dotted line; Cleymans-Suhonen and RGSG models calculations are shown by dashed and dotted lines, respectively. Solid line shows the result of our model.

always lost during the collision due to dissipative processes. In thermal description of the fireball, we ignore the effect of such processes, and we assume that all available kinetic energy (or momentum) is globally thermalized at the freeze-out density. Experimental configuration of the collective flow developed 


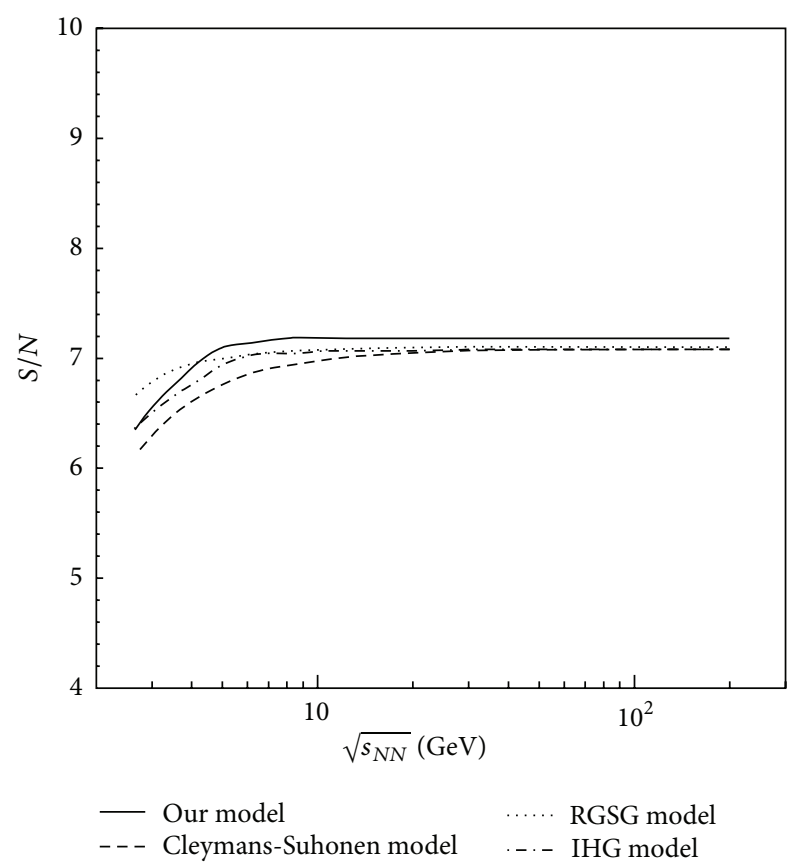

FIGURE 14: Variation of $S / N$ with $\sqrt{s_{N N}}$. IHG model calculation is shown by dash-dotted line; Cleymans-Suhonen and RGSG models calculations are shown by dashed and dotted lines, respectively. Solid line shows the calculation by our model.

in the hot, dense matter reveals the unsatisfactory nature of the above assumption.

4.6. Transport Properties of HG. Transport coefficients are very important tools in quantifying the properties of strongly interacting relativistic fluid and its critical phenomena, that is, phase transition and critical point [127-129]. The fluctuations cause the system to depart from equilibrium and a nonequilibrated system that is created for a brief time. The response of the system to such fluctuations is essentially described by the transport coefficients, for example, shear viscosity, bulk viscosity, speed of sound, and so forth. Recently the data for the collective flow obtained from RHIC and LHC experiments indicate that the system created in these experiments behaves as strongly interacting perfect fluid $[130,131]$, whereas we expected that QGP created in these experiments should behave like a perfect gas. The perfect fluid created after the phase transition indicates a very low value of shear viscosity to entropy ratio so that the dissipative effects are negligible, and the collective flow is large as it was obtained by heavy ion collision experiments [10, 132, 133]. There were several analytic calculations for $\eta$ and $\eta / s$ of simple hadronic systems [134-140] along with some sophisticated microscopic transport model calculations [141-143] in the literature. Furthermore, some calculations predict that the minimum of shear viscosity to entropy density is related with the QCD phase transition [144-149]. Similarly sound velocity is an important property of the matter created in heavy-ion collision experiments because the hydrodynamic evolution of this matter strongly depends on it. A minimum

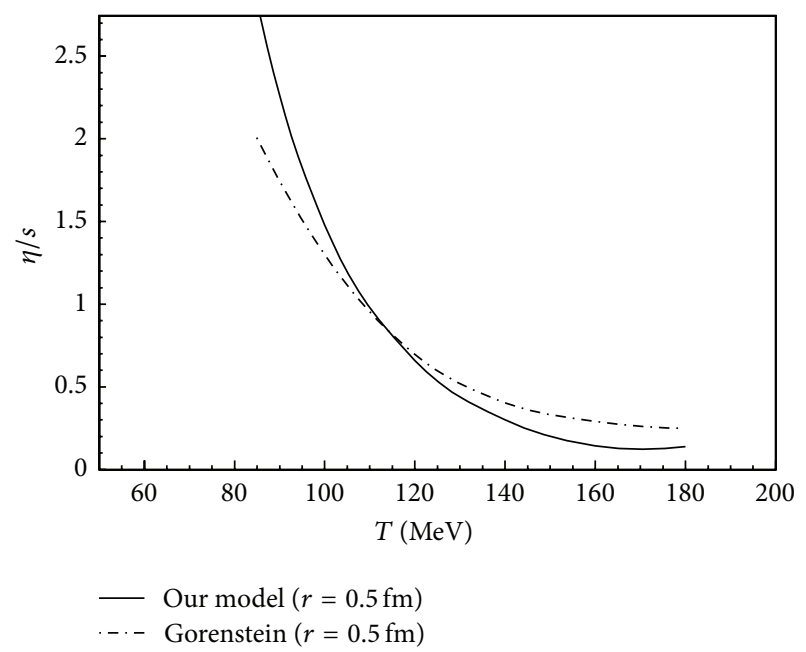

FIGURE 15: Variation of $\eta / s$ with temperature for $\mu_{B}=0$ in our model and a comparison with the results obtained by Gorenstein et al. [157].

occurred in the sound-velocity has also been interpreted in terms of a phase transition [29, 145, 150-155], and further, the presence of a shallow minimum corresponds to a crossover transition [156]. In view of the above, it is worthwhile to study in detail the transport properties of the HG in order to fully comprehend the nature of the matter created in the heavy-ion collisions as well as the involved phase transition phenomenon. In this section, we have used thermal models to calculate the transport properties of HG such as shear viscosity to entropy ratio [31].

We calculate the shear viscosity in our thermal model as it was done previously by Gorenstein et al. [157] using RGSG model. According to molecular kinetic theory, we can write the dependence of the shear viscosity as follows [158]:

$$
\eta \propto \ln \langle|p|\rangle
$$

where $n$ is the particle density, $l$ is the mean free path, and $p$ is the average thermal momentum of the baryons or antibaryons. For the mixture of particle species with different masses and with the same hardcore radius $r$, the shear viscosity can be calculated by the following equation [157]:

$$
\eta=\frac{5}{64 \sqrt{8} r^{2}} \sum_{i}\left\langle\left|p_{i}\right|\right\rangle \times \frac{n_{i}}{n},
$$

where $n_{i}$ is the number density of the ith species of baryons (antibaryons) and $n$ is the total baryon density. In Figure 15, we have shown the variation of $\eta / s$ with respect to temperature as obtained in our model for HG having a baryonic hardcore size $r=0.5 \mathrm{fm}$, and compared our results with those of Gorenstein et al. [157]. We find that near the expected QCD phase transition temperature $\left(T_{c}=170-180 \mathrm{MeV}\right) \eta / s$ shows a lower value in our HG model than the value in other models. In fact, $\eta / s$ in our model looks close to the lower bound $(1 / 4 \pi)$ suggested by AdS/QCD theories $[159,160]$. Recently, measurements in $\mathrm{Pb}-\mathrm{Pb}$ collisions at the large hadron collider (LHC) support the value $\eta / s \approx 1 / 4 \pi$ when compared with the viscous fluid hydrodynamic flow [161]. 


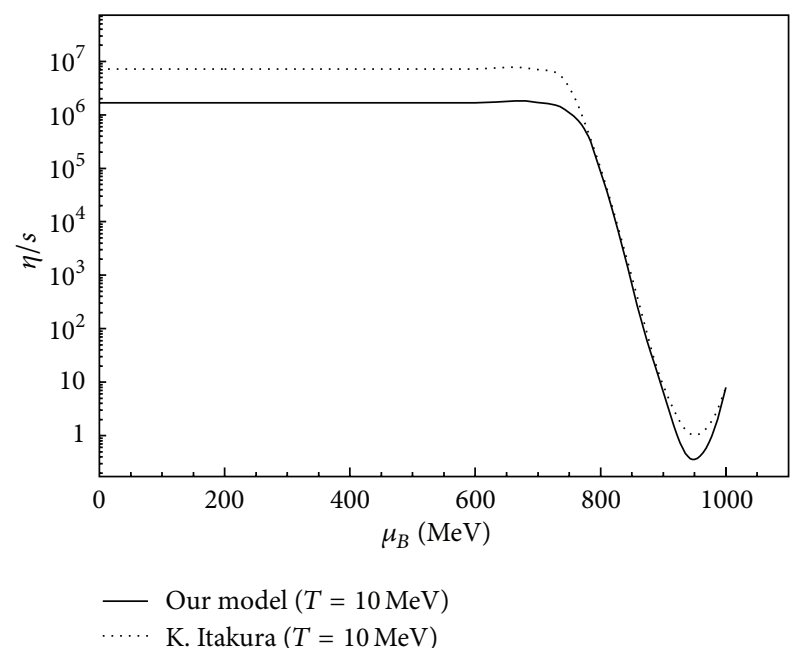

FIGURE 16: Variation of $\eta / s$ with respect to baryon chemical potential $\left(\mu_{B}\right)$ at very low temperature $10 \mathrm{MeV}$. Solid line represents our calculation [31], and dotted curve is for the calculations done by Itakura et al. [139].

In Figure 16, we have shown the variation of $\eta / s$ with respect to $\mu_{B}$ at a very low temperature $(\approx 10 \mathrm{MeV})$ [31]. Here we find that the $\eta / s$ is constant as $\mu_{B}$ increases upto $700 \mathrm{MeV}$ and then sharply decreases. This kind of valley-like structure at low temperature and at $\mu_{B}$ around $950 \mathrm{MeV}$ was also obtained by Chen et al. [137] and Itakura et al. [139]. They have related this structure to the liquid-gas phase transition of the nuclear matter. As we increase the temperature above $20 \mathrm{MeV}$, this valley-like structure disappears. They further suspect that the observation of a discontinuity in the bottom of $\eta / s$ valley may correspond to the location of the critical point. Our HG model yields a curve in complete agreement with these results. Figure 17 represents the variation of $\eta$ and $\eta / s$ with respect to temperature at a fixed $\mu_{B}(=300 \mathrm{MeV})$, for HG having a baryonic hardcore size $r=0.8 \mathrm{fm}$. We have compared our result with the result obtained in [139]. Here we find that $\eta$ increases with temperature in our HG model as well as in the simple phenomenological calculation [139], but it decreases with increasing temperature in lowtemperature effective field theory (EFT) calculations [137, 139]. However, $\eta / s$ decreases with increasing temperature in all three calculations, and $\eta / s$ in our model gives the lowest value at all the temperatures in comparison to other models. In Figure 18, we have shown a comparison between $\eta$ calculated in our HG model with the results obtained in a microscopic pion gas model used in [141]. Our model results show a fair agreement with the microscopic model results for the temperature higher than $160 \mathrm{MeV}$, while at lower temperatures the microscopic calculation predicts lower values of $\eta$ in comparison to our results. The most probable reason may be that the calculations have been done only for pion gas in the microscopic model, while at low temperatures the inclusion of baryons in the HG is very important in order to extract a correct value for the shear viscosity [31]. Figure 19 shows the variation of $\eta / s$ with respect to $\sqrt{s_{N N}}$ in our model calculation. We have compared our results with

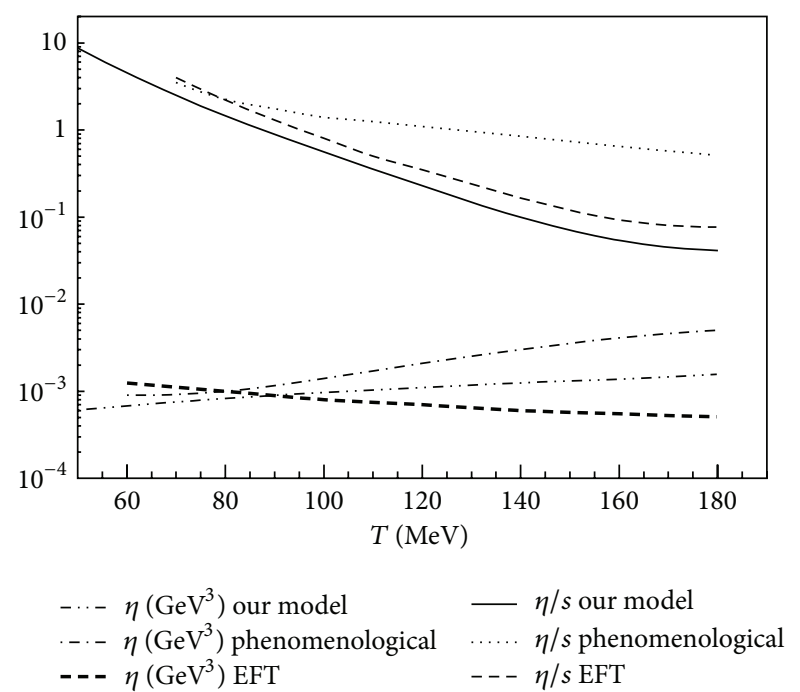

FIGURE 17: Variation of $\eta$ in unit of $(\mathrm{GeV})^{3}$ and $\eta / s$ with respect to temperature at $\mu_{B}=300 \mathrm{MeV}$ in our model [31] and a comparison with the results obtained by Itakura et al. [139].

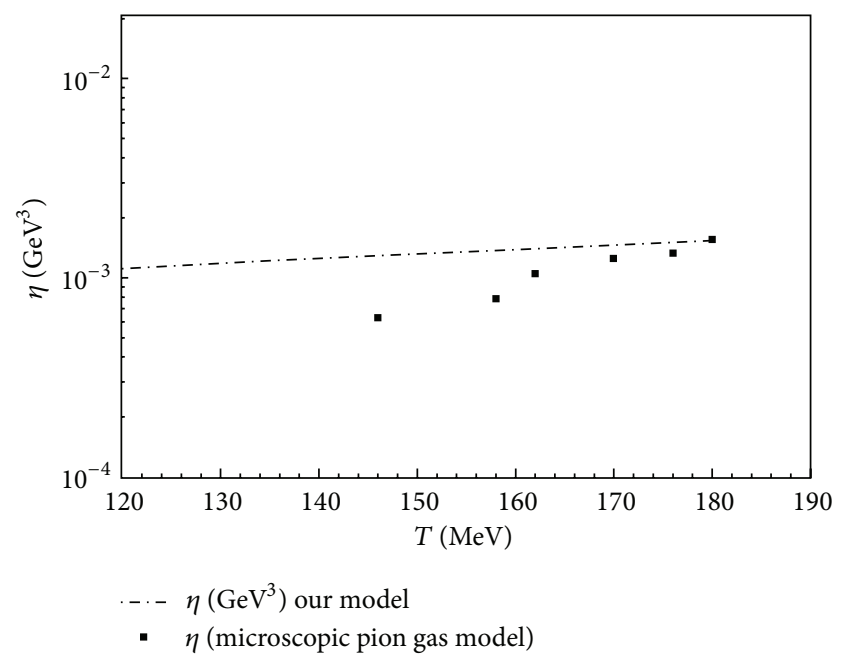

FIGURE 18: Variation of $\eta$ with respect to temperature at $\mu_{B}=$ $300 \mathrm{MeV}$ in our model and a comparison with the results obtained by Muronga [141].

that calculated in [157]. There is similarity in our results at lower energies, while our results significantly differ at higher energies. However both the calculations show that $\eta / s$ decreases with increasing $\sqrt{s_{N N}}$.

The study of the transport properties of nonequilibrium systems which are not far from an equilibrium state has yielded valuable results in the recent past. Large values of the elliptic flow observed at RHIC indicate that the matter in the fireball behaves as a nearly perfect liquid with a small value of the $\eta / s$ ratio. After evaluating $\eta / s$ in strongly coupled theories using AdS/CFT duality conjecture, a lower bound was reported as $\eta / s=1 / 4 \pi$. We surprisingly notice that the fireball with hot, dense HG as described in our model gives transport coefficients which agree with those given in different approaches. Temperature and baryon chemical potential 


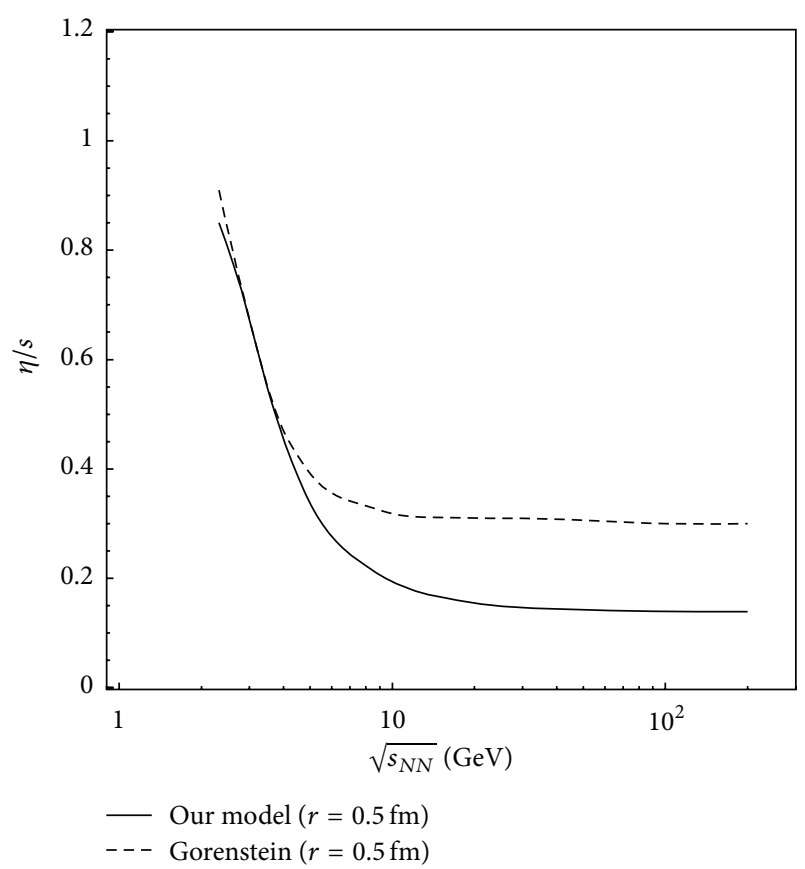

FIGURE 19: Variation of $\eta / s$ with respect to $\sqrt{s_{N N}}$ in our model [31] and other model calculation [157].

dependence of the $\eta / s$ are analyzed and compared with the results obtained in other models. Our results lend support to the claim that knowledge of the EOS and the transport coefficients of HG is essential for a better understanding of the dynamics of the medium formed in the heavy-ion collisions [31].

4.7. Rapidity and Transverse Mass Spectra. In order to suggest any unambiguous signal for QGP, the dynamics of the collisions should be understood properly. Such information can be obtained by analyzing the properties of various particles which are emitted from various stages of the collisions. Hadrons are produced at the end of the hot and dense QGP phase, but they subsequently scatter in the confined hadronic phase prior to decoupling (or "freeze-out") from the collision system, and finally a collective evolution of the hot and dense matter occurs in the form of transverse, radial, or elliptic flow which is instrumental in shaping the important features of the particle spectra. The global properties and dynamics of freeze-out can be at best studied via hadronic observables such as rapidity distributions and transverse mass spectra [162]. There are various approaches for the study of rapidity as well as transverse mass spectra of HG [28, 37, 38, 163-185]. Hadronic spectra from purely thermal models usually reveal an isotropic distribution of particles [186], and hence the rapidity spectra obtained with the purely thermal models do not reproduce the features of the experimental data satisfactorily. Similarly the transverse mass spectra from the thermal models reveal a more steeper curve than that observed experimentally. The comparisons illustrate that the fireball formed in heavy-ion collisions does not expand isotropically in nature, and there is a prominent input of collective flow in the longitudinal and transverse directions which finally causes anisotropy in the rapidity and transverse mass distributions of the hadrons after the freeze-out. Here we mention some kinds of models of thermal and collective flow used in the literature. Hydrodynamical properties of the expanding fireball have been initially discussed by Bjorken and Landau for the central-rapidity and stopping regimes, respectively $[28,163]$. However, collisions even at RHIC energies reveal that they are neither fully stopped, nor fully transparent. As the collision energy increases, the longitudinal flow grows stronger and leads to a cylindrical geometry as postulated in $[37,38,164,165]$. They assume that the fireballs are distributed uniformally in the longitudinal direction and demonstrate that the available data can consistently be described in a thermal model with inputs of chemical equilibrium and flow, although they have also used the experimental data for small systems only. They use two simple parameters: transverse flow velocity $\left(\beta_{r}\right)$ and temperature $(T)$ in their models. In $[166,167]$, nonuniform flow model is used to analyze the spectra specially to reproduce the dip at midrapidity in the rapidity spectra of baryons by assuming that the fireballs are distributed nonuniformly in the longitudinal phase space. In [175-179], the rapidity-dependent baryon chemical potential has been invoked to study the rapidity spectra of hadrons. In certain hydrodynamical models [180], measured transverse momentum $\left(p_{T}\right)$ distributions in Au-Au collisions at $\sqrt{s_{N N}}=$ $130 \mathrm{GeV}$ [181-183] have been described successfully by incorporating a radial flow. In [184], rapidity spectra of mesons have been studied using viscous relativistic hydrodynamics in a $1+1$ dimension assuming a nonboost invariant Bjorken's flow in the longitudinal direction. They have also analyzed the effect of the shear viscosity on the longitudinal expansion of the matter. Shear viscosity counteracts the gradients of the velocity field; as a consequence it slows down the longitudinal expansion. Ivanov [185] has employed 3 FD model [187] for the study of rapidity distributions of hadrons in the energy range from $2.7 \mathrm{GeV}$ to $62.4 \mathrm{GeV}$. In $3 \mathrm{FD}$ model, three different EOS: (i) a purely hadronic EOS, (ii) the EOS involving first order phase transition from hot, dense HG to QGP, and (iii) the EOS with smooth crossover transition are used. Within all three scenarios they reproduced the data at the almost same extent. In [188], rapidity distributions of various hadrons in the central nucleus-nucleus collisions have been studied in the Landau's and Bjorken's hydrodynamical model. The effect of speed of sound $\left(c_{s}\right)$ on the hadronic spectra and the correlation of $c_{s}$ with freeze-out parameters are indicated.

In this section, we study the rapidity and transverse mass spectra of hadrons using thermal approach. We can rewrite (36) in the following manner [58]:

$$
\begin{aligned}
n_{i}^{\mathrm{ex}}=\frac{g_{i} \lambda_{i}}{(2 \pi)^{3}}[ & \left((1-R)-\lambda_{i} \frac{\partial R}{\partial \lambda_{i}}\right) \int_{0}^{\infty} \frac{d^{3} k}{\left[\exp \left(E_{i} / T\right)+\lambda_{i}\right]} \\
& \left.-\lambda_{i}(1-R) \int_{0}^{\infty} \frac{d^{3} k}{\left[\exp \left(E_{i} / T\right)+\lambda_{i}\right]^{2}}\right] .
\end{aligned}
$$


It means that the invariant distributions are $[37,38,164]$

$$
\begin{aligned}
E_{i} \frac{d^{3} N_{i}}{d k^{3}}=\frac{g_{i} V \lambda_{i}}{(2 \pi)^{3}}[ & \left((1-R)-\lambda_{i} \frac{\partial R}{\partial \lambda_{i}}\right) \frac{E_{i}}{\left[\exp \left(E_{i} / T\right)+\lambda_{i}\right]} \\
& \left.-\lambda_{i}(1-R) \frac{E_{i}}{\left[\exp \left(E_{i} / T\right)+\lambda_{i}\right]^{2}}\right] .
\end{aligned}
$$

If we use Boltzmann's approximation, (63) differs from the one used in the paper of Schnedermann et al. [164] by the presence of a prefactor $\left[(1-R)-\lambda_{i} \partial R / \partial \lambda_{i}\right]$. However, we measure all these quantities precisely at the chemical freeze-out using our model, and hence quantitatively we do not require any normalizing factor as required in [164]. We use the following expression to calculate the rapidity distributions of baryons in the thermal model [58]:

$$
\begin{aligned}
\left(\frac{d N_{i}}{d y}\right)_{\text {th }}=\frac{g_{i} V \lambda_{i}}{\left(2 \pi^{2}\right)} \quad[ & \left.(1-R)-\lambda_{i} \frac{\partial R}{\partial \lambda_{i}}\right) \\
& \times \int_{0}^{\infty} \frac{m_{T}^{2} \cosh y d m_{T}}{\left[\exp \left(m_{T} \cosh y / T\right)+\lambda_{i}\right]} \\
& -\lambda_{i}(1-R) \\
& \left.\times \int_{0}^{\infty} \frac{m_{T}^{2} \cosh y d m_{T}}{\left[\exp \left(m_{T} \cosh y / T\right)+\lambda_{i}\right]^{2}}\right] .
\end{aligned}
$$

Here $y$ is the rapidity variable and $m_{T}$ is the transverse mass $\left(m_{T}=\sqrt{m^{2}+p_{T}^{2}}\right)$. Also $V$ is the total volume of the fireball formed at chemical freeze-out, and $N_{i}$ is the total number of $i$ th baryons. We assume that the freeze-out volume of the fireball for all types of hadrons at the time of the homogeneous emissions of hadrons remains the same. It can be mentioned here that in the above equation, there occurs no free parameter because all the quantities $g, V, \lambda, R$, and so forth, are determined in the model. However, (64) describes the experimental data only at midrapidity, while it fails at forward and backward rapidities, so we need to modify it by incorporating a flow factor in the longitudinal direction. Thus the resulting rapidity spectra of ith hadron is $[37,38,58,164]$

$$
\frac{d N_{i}}{d y}=\int_{-\eta_{\max }}^{\eta_{\max }}\left(\frac{d N_{i}}{d y}\right)_{\mathrm{th}}(y-\eta) d \eta
$$

where $\left(d N_{i} / d y\right)_{\text {th }}$ can be calculated by using (64). The expression for average longitudinal velocity is $[166,167,189]$

$$
\left\langle\beta_{L}\right\rangle=\tanh \left(\frac{\eta_{\max }}{2}\right) \text {. }
$$

Here $\eta_{\max }$ is a free parameter which provides the upper rapidity limit for the longitudinal flow velocity at a particular $\sqrt{s_{N N}}$, and its value is determined by the best experimental fit. The value of $\eta_{\max }$ increases with the increasing $\sqrt{s_{N N}}$, and hence $\beta_{L}$ also increases. Cleymans et al. [179] have extended the thermal model $[175,176]$, in which the chemical freeze-out parameters are rapidity-dependent, to calculate the rapidity spectra of hadrons. They use the following expression for rapidity spectra:

$$
\frac{d N^{i}}{d y}=\int_{-\infty}^{+\infty} \rho\left(y_{\mathrm{FB}}\right) \frac{d N_{1}^{i}\left(y-y_{\mathrm{FB}}\right)}{d y} d y_{\mathrm{FB}}
$$

where $d N_{1}^{i} / d y$ is the thermal rapidity distribution of particles calculated by using (64) and $\rho\left(y_{\mathrm{FB}}\right)$ is a Gaussian distribution of fireballs centered at zero and given by

$$
\rho\left(y_{\mathrm{FB}}\right)=\frac{1}{\sqrt{2 \pi} \sigma} \exp \left(\frac{-y_{\mathrm{FB}}^{2}}{2 \sigma^{2}}\right) \text {. }
$$

Similarly we calculate the transverse mass spectra of hadrons by using following expression [58]:

$$
\frac{d N_{i}}{m_{T} d m_{T}}=\frac{g_{i} V \lambda_{i}}{\left(2 \pi^{2}\right)}\left[(1-R)-\lambda_{i} \frac{\partial R}{\partial \lambda_{i}}\right] m_{T} K_{1}\left(\frac{m_{T}}{T}\right),
$$

where $K_{1}\left(m_{T} / T\right)$ is the modified Bessel's function:

$$
K_{1}\left(\frac{m_{T}}{T}\right)=\int_{0}^{\infty} \cosh y\left[\exp \left(\frac{-m_{T} \cosh y}{T}\right)\right] d y .
$$

The above expression for transverse mass spectra arises from a stationary thermal source alone which is not capable of describing the experimental data successfully. So, we incorporate flow velocity in both the directions in (69), longitudinal as well as transverse, in order to describe the experimental data satisfactorily. After defining the flow velocity field, we can calculate the invariant momentum spectrum by using the following formula $[164,190]$ :

$$
\begin{aligned}
E_{i} \frac{d^{3} N_{i}}{d k^{3}}= & \frac{g_{i} V \lambda_{i}}{(2 \pi)^{3}}\left[(1-R)-\lambda_{i} \frac{\partial R}{\partial \lambda_{i}}\right] \\
& \times \int \exp \left(\frac{-k_{\mu} u^{\mu}}{T}\right) k_{\lambda} d \sigma_{\lambda} .
\end{aligned}
$$

While deriving (71), we assume that the local fluid velocity $u^{\mu}$ gives a boost to an isotropic thermal distribution of hadrons. Now the final expression of transverse mass spectra of hadrons after incorporation of flow velocity in our model is [58]

$$
\begin{aligned}
\frac{d N_{i}}{m_{T} d m_{T}}= & \frac{g_{i} V \lambda_{i} m_{T}}{\left(2 \pi^{2}\right)}\left[(1-R)-\lambda_{i} \frac{\partial R}{\partial \lambda_{i}}\right] \\
& \times \int_{0}^{R_{0}} r d r K_{1}\left(\frac{m_{T} \cosh \rho}{T}\right) I_{0}\left(\frac{p_{T} \sinh \rho}{T}\right) .
\end{aligned}
$$

Here $I_{0}\left(p_{T} \sinh \rho / T\right)$ is the modified Bessel's function:

$$
I_{0}\left(\frac{p_{T} \sinh \rho}{T}\right)=\frac{1}{2 \pi} \int_{0}^{2 \pi} \exp \left(\frac{p_{T} \sinh \rho \cos \phi}{T}\right) d \phi,
$$

where $\rho$ is given by $\rho=\tanh ^{-1} \beta_{r}$, with the velocity profile chosen as $\beta_{r}=\beta_{s}(\xi)^{n}[37,38,164] . \beta_{s}$ is the maximum surface 


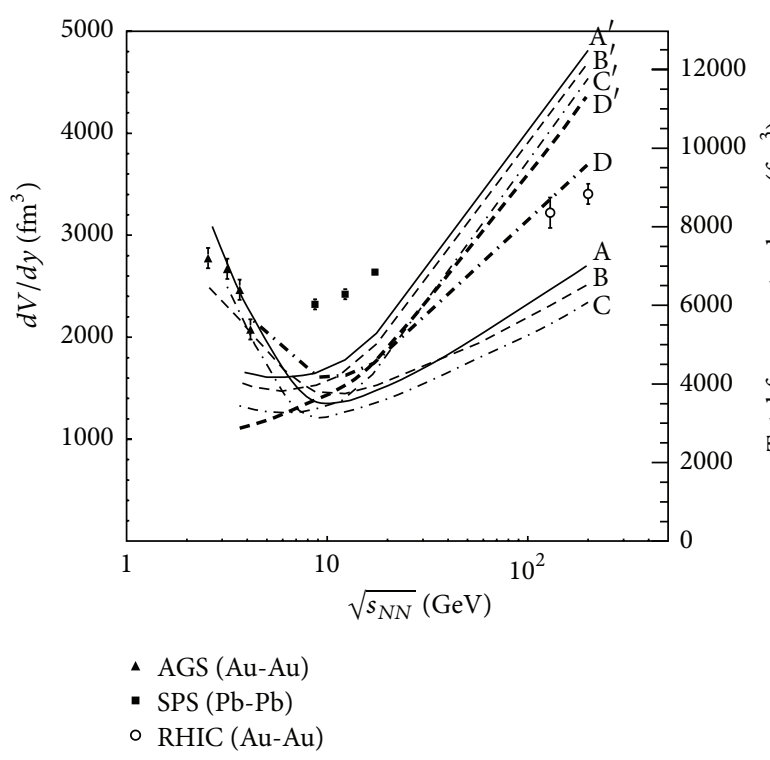

Figure 20: Energy dependence of the freeze-out volume for the central nucleus-nucleus collisions. The symbols are the HBT data for freeze-out volume $V_{\mathrm{HBT}}$ for the $\pi^{+}$[191]. $A^{\prime}, B^{\prime}$, and $C^{\prime}$ are the total freeze-out volume and $A, B$, and $C$ depict the $d V / d y$ as found in our model for $\pi^{+}, K^{+}$and $K^{-}$, respectively. $D$ represents the total freeze-out volume for $\pi^{+}$calculated in the Ideal HG model. $D^{\prime}$ is the the total freeze-out volume for $\pi^{+}$in our model calculation using Boltzmann's statistics [58].

velocity and is treated as a free parameter and $\xi=\left(r / R_{0}\right)$. The average of the transverse velocity can be evaluated as [182]

$$
\left\langle\beta_{r}\right\rangle=\frac{\int \beta_{s} \xi^{n} \xi d \xi}{\int \xi d \xi}=\left(\frac{2}{2+n}\right) \beta_{s}
$$

In our calculation, we use a linear velocity profile $(n=$ 1 ), and $R_{0}$ is the maximum radius of the expanding source at freeze-out $(0<\xi<1)$ [182].

In Figure 20, we have shown the variations of $V$ and $d V / d y$ with the $\sqrt{s_{N N}}$ calculated in our excluded-volume model and compared with the results of various thermal models. We show the total freeze-out volume for $\pi^{+}$calculated in our model using Boltzmann's statistics. We see that there is a significant difference between the results arising from quantum statistics and Boltzmann's statistics [58]. We also show the total freeze-out volume for $\pi^{+}$in IHG model calculation by dash-dotted line $D$. We clearly notice a remarkable difference between the results of our excluded-volume model and those of IHG model also. We have also compared predictions from our model with the data obtained from the pion interferometry (HBT) [191] which in fact reveals thermal (kinetic) freeze-out volumes. The results of thermal models support the finding that the decoupling of strange mesons from the fireball takes place earlier than the $\pi$-mesons. Moreover, a flat minimum occurs in the curves around the center-of-mass energy $\approx 8 \mathrm{GeV}$, and this feature is well supported by HBT data. In Figure 21, we present the rapidity distribution of $\pi^{+}$for central $\mathrm{Au}+\mathrm{Au}$ collisions at $\sqrt{s_{N N}}=200 \mathrm{GeV}$ over full rapidity range. Dotted line shows

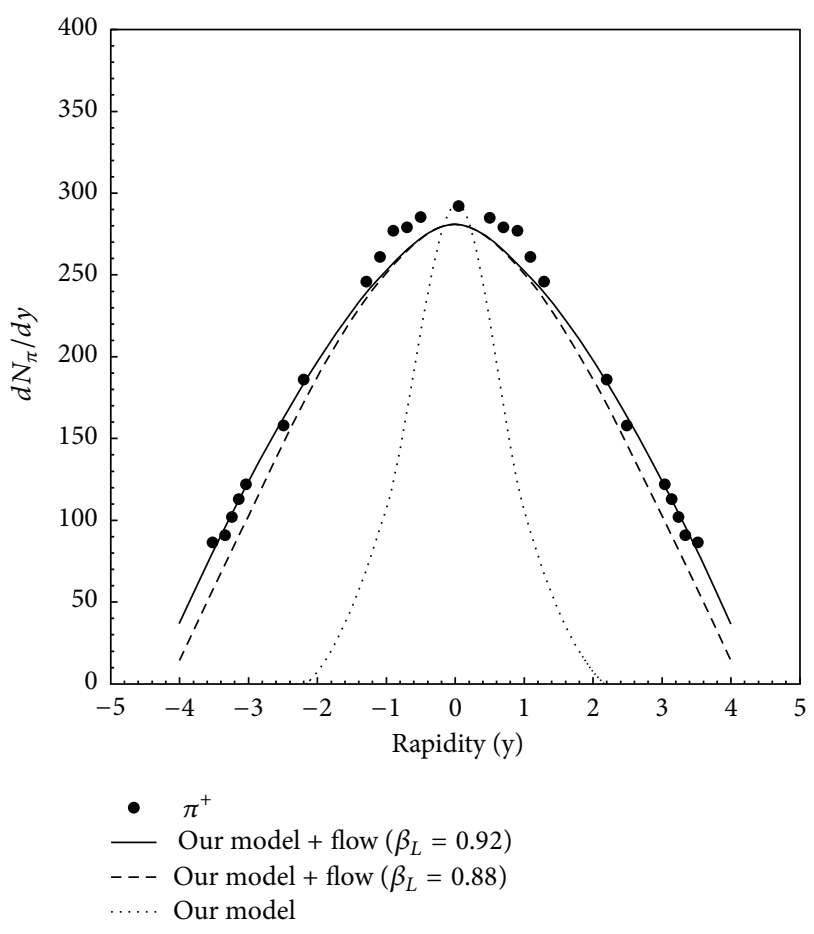

FIgURE 21: Rapidity distribution of $\pi^{+}$at $\sqrt{s_{N N}}=200 \mathrm{GeV}$. Dotted line shows the rapidity distribution calculated in our thermal model [58]. Solid line and dashed line show the results obtained after incorporating longitudinal flow in our thermal model. Symbols are the experimental data [192].

the distribution of $\pi^{+}$due to purely thermal model. Solid line shows the rapidity distributions of $\pi^{+}$after the incorporation of longitudinal flow in our thermal model, and results give a good agreement with the experimental data [192]. In fitting the experimental data, we use the value of $\eta_{\max }=3.2$ and hence the longitudinal flow velocity $\beta_{L}=0.92$ at $\sqrt{s_{N N}}=$ $200 \mathrm{GeV}$. For comparison and testing the appropriateness of this parameter, we also show the rapidity distributions at a different value, that is, $\eta_{\max }=2.8$ (or, $\beta_{L}=0.88$ ), by a dashed line in the figure. We find that the results slightly differ, and hence it shows a small dependence on $\eta_{\max }$ [58]. Figure 22 represents the rapidity distributions of pion at various $\sqrt{s_{N N}}$ calculated by using (67) [179]. There is a good agreement between the model results and experimental data at all $\sqrt{s_{N N}}$.

In Figure 23, we show the transverse mass spectra for $\pi^{+}$and proton for the most central collisions of $\mathrm{Au}+\mathrm{Au}$ at $\sqrt{s_{N N}}=200 \mathrm{GeV}$. We have neglected the contributions from the resonance decays in our calculations since these contributions affect the transverse mass spectra only towards the lower transverse mass side, that is, $m_{T}<0.3 \mathrm{GeV}$. We find a good agreement between our calculations and the experimental results for all $m_{T}$ except $m_{T}<0.3 \mathrm{GeV}$ after incorporating the flow velocity in purely thermal model. This again shows the importance of collective flow in the description of the experimental data [193]. At this energy, the value of $\beta_{s}$ is taken as 0.50 and transverse flow velocity $\beta_{r}=0.33$. This set of transverse flow velocity is able to reproduce the transverse mass spectra of almost all the 


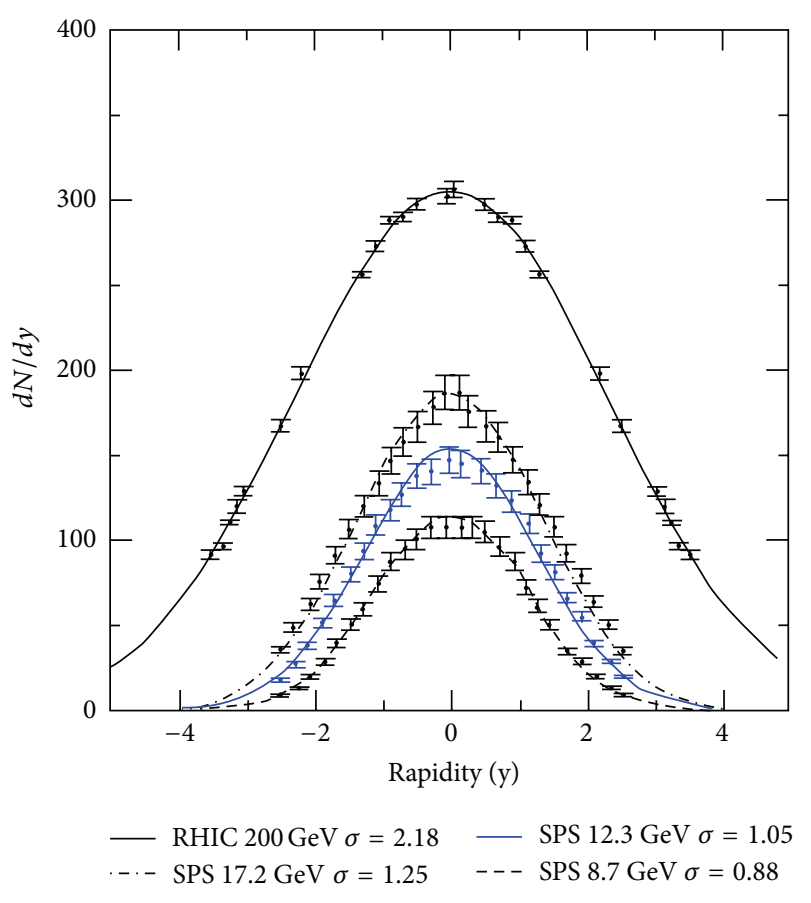

FIGURE 22: Rapidity distribution of pion at various $\sqrt{s_{N N}}$. Lines are model results and points are experimental data. Figure 22 is taken from [179].

hadrons at $\sqrt{s_{N N}}=200 \mathrm{GeV}$. We notice that the transverse flow velocity slowly increases with the increasing $\sqrt{s_{N N}}$. If we take $\beta_{s}=0.60$, we find that the results differ with data as shown in Figure 23. In Figure 24, we show the transverse momentum $\left(p_{T}\right)$ spectra for $\pi^{+}, K^{+}$, and $p$ in the most central collisions of Au-Au at $\sqrt{s_{N N}}=200 \mathrm{GeV}$. Our model calculations reveal a close agreement with the experimental data [193]. In Figure 25, we show the $p_{T}$ spectra of $\pi^{-}, K^{-}$, and $\bar{p}$ for the $\mathrm{Pb}-\mathrm{Pb}$ collisions at $\sqrt{s_{N N}}=2.76 \mathrm{TeV}$ at the LHC. Our calculations again give a good fit to the experimental results [194]. We also compare our results for $\bar{p}$ spectrum with the hydrodynamical model of Shen et al. [195], which successfully explains $\pi^{-}$and $K^{-}$spectra but strongly fails in the case of $\bar{p}$ spectrum [58]. In comparison, our model results show closer agreement with the experimental data. Shen et al. [195] have employed (2+1)-dimensional viscous hydrodynamics with the lattice QCD-based EOS. They use Cooper-Frye prescription to implement kinetic freeze-out in converting the hydrodynamic output into the particle spectra. Due to lack of a proper theoretical and phenomenological knowledge, they use the same parameters for $\mathrm{Pb}-\mathrm{Pb}$ collisions at LHC energy, which was used for $\mathrm{Au}-\mathrm{Au}$ collisions at $\sqrt{s_{N N}}=200 \mathrm{GeV}$. Furthermore, they use the temperatureindependent $\eta / s$ ratio in their calculation. After fitting the experimental data, we get $\beta_{s}=0.80\left(\beta_{r}=0.53\right)$ at this energy which indicates the collective flow as becoming stronger at LHC energy than that observed at RHIC energies. In this plot, we also attempt to show how the spectra for $\pi^{-}$will change at a slightly different value of the parameter, that is, $\beta_{s}=0.88$ [58].

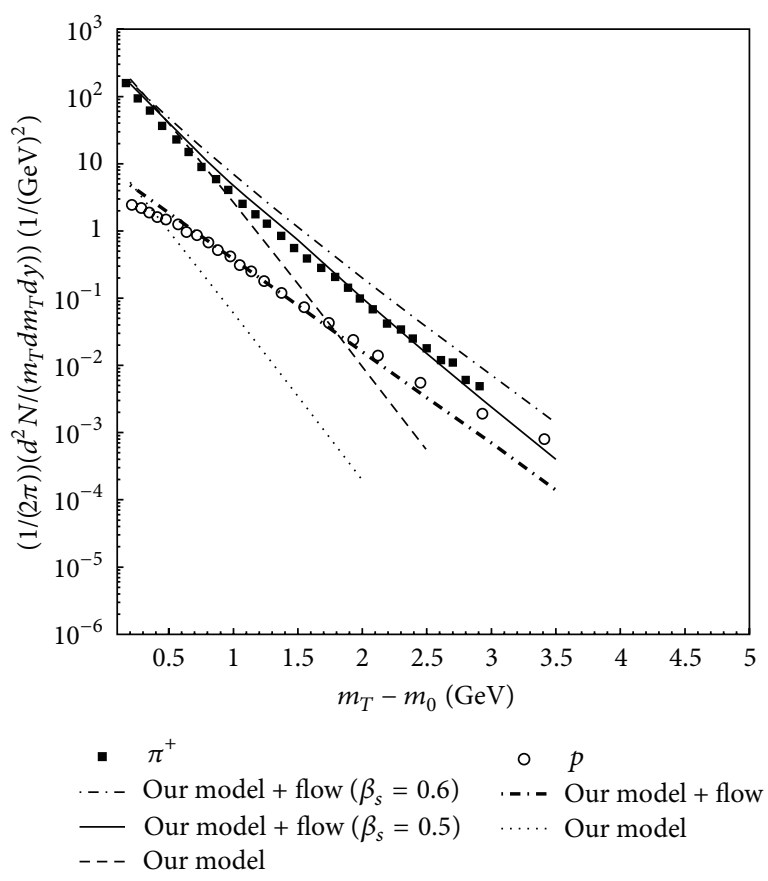

Figure 23: Transverse mass spectra for $\pi^{+}$and proton for the most central collisions at $\sqrt{s_{N N}}=200 \mathrm{GeV}$. Dashed and dotted lines are the transverse mass spectra due to purely thermal source for $\pi^{+}$and proton, respectively. Solid and dash-dotted lines are the results for $\pi^{+}$and proton, respectively, obtained after incorporation of flow in thermal model [58]. Symbols are the experimental data [193].

\section{Summary and Conclusions}

The main aim in this paper is to emphasize the use of the thermal approach in describing various yields of different particle species that have been measured in various experiments running at various places. We have discussed various types of thermal approaches for the formulation of EOS for HG. We have argued that, incorporation of interactions between hadrons in a thermodynamically consistent way is important for the realistic formulation of HG from both qualitatively and quantitatively point of view. We have presented the systematic study of the particle production in heavy-ion collisions from AGS to LHC energy. We have observed from this analysis that the production of the particles seems to occurr according to principle of equilibrium. Yields of hadrons and their ratios measured in heavy-ion collisions match with the predictions of thermal models assured the thermalization of the collision fireball formed in heavy-ion collisions. Furthermore, various experimental observables such as transverse momentum spectra and elliptic flow indicate the presence of the thermodynamical pressure, developed in the early stage, and correlations which are expected in a thermalized medium.

We have discussed a detailed formulation of various excluded-volume models and their shortcomings. Some excluded-volume models are not thermodynamically consistent because they do not possess a well defined partition function from which various thermodynamical quantities 


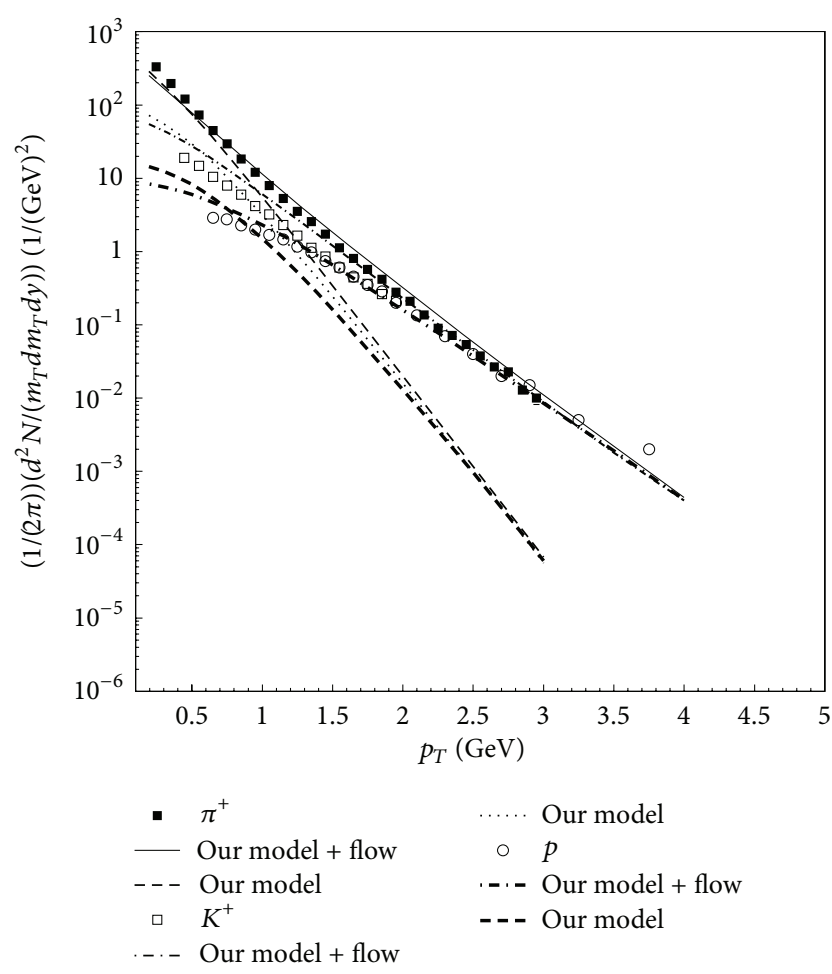

Figure 24: Transverse momentum spectra for $\pi^{+}, p$, and $K^{+}$for the most central Au-Au collision at $\sqrt{s_{N N}}=200 \mathrm{GeV}$ [58]. Lines are the results of our model calculation, and symbols are the experimental results [193].

such as number density can be calculated. However, some of them are the thermodynamically consistent but suffer from some unphysical situations cropping up in the calculations. We have proposed a new approximately thermodynamically consistent excluded-volume model for a hot and dense HG. We have used quantum statistics in the grand canonical partition function of our model so that it works even at extreme values of $T$ and $\mu_{B}$ where all other models fail. Moreover, our model respects causality. We have presented the calculations of various thermodynamical quantities such as entropy per baryon and energy density in various excludedvolume models and compare the results with those of a microscopic approach URASiMA. We find that our model results are in close agreement with that of the entirely different approach URASiMA model. We have calculated various particle ratios at various $\sqrt{s_{N N}}$, and we confronted the results of various thermal models with the experimental data and find that they are indeed successful in describing the particle ratios. However, we find that our model results are closer to the experimental data in comparison to those of other excluded-volume models. We have calculated some conditions such as $E / N$ and $S / N$ at chemical freeze-out points and attempted to test whether these conditions involve energy independence as well as independence of structure of the nuclei involved in the collisions. We find that $E / N \approx$ $1.0 \mathrm{GeV}$ and $S / N \approx 7.0$ are the two robust freeze-out criteria which show independence of the energy and structure of nuclei. Moreover, the calculations of transport properties in

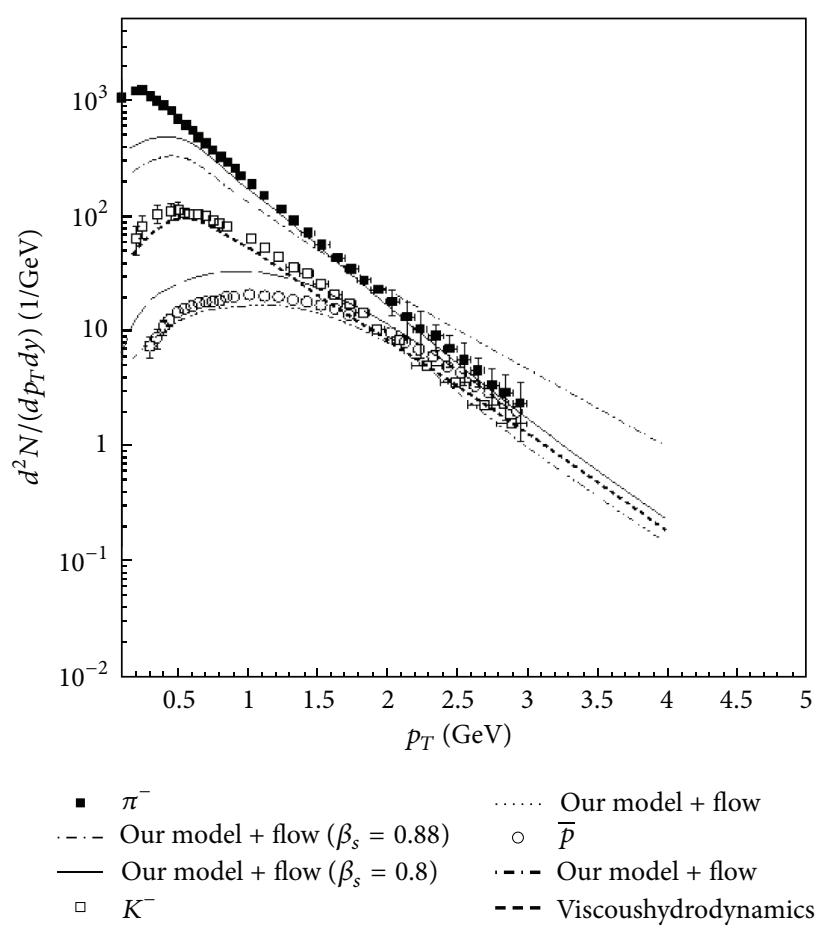

FIgURE 25: Transverse momentum spectra of various hadrons for the most central collisions of $\mathrm{Pb}-\mathrm{Pb}$ at $\sqrt{s_{N N}}=2.76 \mathrm{TeV}$ from LHC [58]. Lines are the results of model calculations, and symbols are the experimental results [194]. Thick-dashed line is the prediction of viscous-hydrodynamical model [195] for $\bar{p}$.

our model match well with the results obtained in other widely different approaches. Further, we present an analysis of rapidity distributions and transverse mass spectra of hadrons in central nucleus-nucleus collision at various $\sqrt{s_{N N}}$ using our EOS for HG. We see that the stationary thermal source alone cannot describe the experimental data fully unless we incorporate flow velocities in the longitudinal as well as in the transverse direction, and as a result, our modified model predictions show a good agreement with the experimental data. Our analysis shows that a collective flow develops at each $\sqrt{s_{N N}}$ which increases further with the increasing $\sqrt{s_{N N}}$. The description of the rapidity distributions and transverse mass spectra of hadrons at each $\sqrt{s_{N N}}$ matches very well with the experimental data. Thus, we emphasize that thermal models are indeed an important tool to describe the various features of hot and dense HG. Although, these models are not capable of telling whether QGP was formed before HG phase, they can give an indirect indication of it by showing any anomalous feature as observed in the experimental data.

In conclusion, the net outcome of this review is indeed a surprising one. The excluded-volume HG models are really successful in describing all kinds of features of the HG formed in ultrarelativistic heavy-ion collisions. The most important property indicated by such description is the chemical equilibrium reached in such collisions. However, the description is still a geometrical one and does not involve any microscopic picture of interactions. Moreover, its relativistic and field theoretic generalizations are still needed 
in order to make the picture a more realistic description. But it is amazing to find that these models still work much better than expected. Notwithstanding these remarks, we should add that Lattice QCD results are now available for the pressure, entropy density, and energy density, and so forth for the entire temperature range from $T=0$ to higher values at $\mu=0$. Here low temperature phase of QCD is the HG, and recently our excluded-volume model reproduces these properties quite in agreement with Lattice results [196]. We have also used these calculations in the precise determination of the QCD critical end point [197, 198]. Thus we conclude that the excluded-volume models are successful in reproducing numerical results obtained in various experiments, and, therefore, further research is required to show how these descriptions are connected with the microscopic interactions.

\section{Acknowledgment}

S. K. Tiwari is grateful to the Council of Scientific and Industrial Research (CSIR), New Delhi, for providing a research grant.

\section{References}

[1] C. P. Singh, "Signals of quark-gluon plasma," Physics Report, vol. 236, no. 3, pp. 147-224, 1993.

[2] C. P. Singh, "current status of properties and signals of the quark-gluon plasma," International Journal of Modern Physics A, vol. 7, p. 7185, 1992.

[3] M. J. Tannenbaum, "Recent results in relativistic heavy ion collisions: from "a new state of matter" to "the perfect fluid'”, Reports on Progress in Physics, vol. 69, p. 2005, 2006.

[4] B. Müller, "Physics and signatures of the quark-gluon plasma," Reports on Progress in Physics, vol. 58, p. 611, 1995.

[5] H. Satz, "Colour deconfinement in nuclear collisions," Reports on Progress in Physics, vol. 63, no. 9, pp. 1511-1574, 2000.

[6] H. Satz, "quark matter and nuclear collisions a brief history of strong interaction thermodynamics," International Journal of Modern Physics E, vol. 21, Article ID 1230006, 23 pages, 2012.

[7] P. Braun-Munzinger, K. Redlich, and J. Stachel, Invited Review for Quark Gluon Plasma, vol. 3, Edited by R. C. Hwa and X.-N. Wang, World Scientific Publishing.

[8] P. Braun-Munzinger and J. Wambach, "Colloquiumml: phase diagram of strongly interacting matter," Reviews of Modern Physics, vol. 81, no. 3, pp. 1031-1050, 2009.

[9] P. Braun-Munzinger and J. Stachel, “The quest for the quarkgluon plasma," Nature, vol. 448, no. 7151, pp. 302-309, 2007.

[10] M. Gyulassy and L. McLerran, "New forms of QCD matter discovered at RHIC," Nuclear Physics A, vol. 750, no. 1, pp. 3063, 2005.

[11] K. Adcoxbd, S.S. Adlere, S. Afanasiev et al., "Formation of dense partonic matter in relativistic nucleus-nucleus collisions at RHIC: experimental evaluation by the PHENIX Collaboration," Nuclear Physics A, vol. 757, pp. 184-283, 2005.

[12] R. Rapp, "Signatures of thermal dilepton radiation at ultrarelativistic energies," Physical Review C, vol. 63, Article ID 054907, 13 pages, 2001.
[13] V. Ruuskanen, "transverse hydrodynamics with a first order phase transition in very high-energy nuclear collisions," Acta Physica Polonica B, vol. 18, p. 551, 1987.

[14] I. Krasnikova, C. Gale, and D. K. Srivastava, "Production of intermediate mass dileptons in relativistic heavy ion collisions," Physical Review C, vol. 65, Article ID 064903, 2002.

[15] W. Cassing, E. L. Bratkovskaya, R. Rapp, and J. Wambach, "Probing the $\rho$ spectral function in hot and dense nuclear matter by dileptons," Physical Review C, vol. 57, no. 2, pp. 916-921, 1998.

[16] J. Wambach and R. Rapp, "Theoretical interpretations of lowmass dileptons," Nuclear Physics A, vol. 638, no. 1-2, p. 171, 1998.

[17] K. Gallmeister, B. Kämpfer, and O. P. Pavlenko, "Is there a unique thermal source of dileptons in $\mathrm{Pb}(158 \mathrm{~A} \cdot \mathrm{GeV})+\mathrm{Au}, \mathrm{Pb}$ reactions?” Physics Letters B, vol. 473, no. 1-2, pp. 20-24, 2000.

[18] F. Karsch, K. Redlich, and L. Turko, "Chiral symmetry and dileptons in heavy ion collisions," Zeitschrift für Physik C, vol. 60, no. 3, pp. 519-525, 1993.

[19] K. Kajantie, J. Kapusta, L. McLerran, and A. Mekjian, "Dilepton emission and the QCD phase transition in ultrarelativistic nuclear collisions," Physical Review D, vol. 34, no. 9, pp. 2746$2754,1986$.

[20] T. Hatsuda, "Spectral change of hadrons and chiral symmetry," Nuclear Physics A, vol. 698, p. 243, 2002.

[21] K. Yokokawa, T. Hatsuda, A. Hayashigaki, and T. Kunihiro, "Simultaneous softening of $\sigma$ and $\rho$ mesons associated with chiral restoration," Physical Review C, vol. 66, Article ID 022201, 2002.

[22] U. Heinz, "The Little Bang: searching for quark-gluon matter in relativistic heavy-ion collisions," Nuclear Physics A, vol. 685, pp. 414-431, 2001.

[23] P. F. Kolb, J. Sollfrank, P. V. Ruuskanen, and U. Heinz, "Hydrodynamic simulation of elliptic flow," Nuclear Physics A, vol. 661, p. $349,1999$.

[24] D. Teaney, J. Lauret, and E. V. Shuryak, "Flow at the SPS and RHIC as a quark-gluon plasma signature," Physical Review Letters, vol. 86, no. 21, pp. 4783-4786, 2001.

[25] U. A. Wiedemann and U. Heinz, "Particle interferometry for relativistic heavy-ion collisions," Physics Report, vol. 319, no. 4-5, pp. 145-230, 1999.

[26] B. Tomasik, U. A. Wiedemann, and U. Heinz, "Dynamics and sizes of the fireball at freeze-out," Nuclear Physics A, vol. 663, pp. 753-756, 2000.

[27] J. R. Nix, "Low freeze-out temperature and high collective velocities in relativistic heavy-ion collisions," Physical Review C, vol. 58, no. 4, pp. 2303-2310, 1998.

[28] J. D. Bjorken, "Highly relativistic nucleus-nucleus collisions: the central rapidity region," Physical Review D, vol. 27, no. 1, pp. 140$151,1983$.

[29] P. Braun-Munzinger and J. Stachel, "Probing the phase boundary between hadronic matter and the quark-gluon plasma in relativistic heavy-ion collisions," Nuclear Physics A, vol. 606, no. 1-2, pp. 320-328, 1996.

[30] A. Andronic, P. Braun-Munzinger, and J. Stachel, "Hadron production in central nucleus-nucleus collisions at chemical freeze-out," Nuclear Physics A, vol. 772, no. 3-4, pp. 167-199, 2006.

[31] S. K. Tiwari, P. K. Srivastava, and C. P. Singh, "Description of hot and dense hadron-gas properties in a new excluded-volume model," Physical Review C, vol. 85, no. 1, Article ID 014908, 2012.

[32] M. Mishra and C. P. Singh, "Effect of geometrical size of the particles in a hot and dense hadron gas," Physical Review C, vol. 76, Article ID 024908, 9 pages, 2007. 
[33] M. Mishra and C. P. Singh, "Particle multiplicities and ratios in excluded volume models," Physical Review C, vol. 78, Article ID 024910, 9 pages, 2008.

[34] J. Cleymans and K. Redlich, "Unified description of freeze-out parameters in relativistic heavy ion collisions," Physical Review Letters, vol. 81, no. 24, pp. 5284-5286, 1998.

[35] P. Braun-Munzinger, D. Magestro, K. Redlich, and J. Stachel, "Hadron production in Au-Au collisions at RHIC," Physics Letters B, vol. 518, no. 1-2, pp. 41-46, 2001.

[36] D. Magestro, "Evidence for chemical equilibration at RHIC," Journal of Physics G, vol. 28, no. 7, pp. 1745-1752, 2002.

[37] P. Braun-Munzinger, J. Stachel, J. P. Wessels, and N. Xu, “Thermal equilibration and expansion in nucleus-nucleus collisions at the AGS," Physics Letters B, vol. 344, no. 1-4, pp. 43-48, 1995.

[38] P. Braun-Munzinger, J. Stachel, J.P. Wessels, and N. Xu, “Thermal and hadrochemical equilibration in nucleus-nucleus collisions at the SPS," Physics Letters B, vol. 365, pp. 1-6, 1996.

[39] P. Braun-Munzinger, I. Heppe, and J. Stachel, "Chemical equilibration in $\mathrm{Pb}+\mathrm{Pb}$ collisions at the SPS," Physics Letters B, vol. 465, no. 1-4, pp. 15-20, 1999.

[40] S. V. Akkelin, P. Braun-Munzinger, and Y. M. Sinyukov, "Reconstruction of hadronization stage in $\mathrm{Pb}+\mathrm{Pb}$ collisions at $158 \mathrm{~A}$ GeV/c," Nuclear Physics A, vol. 710, no. 3-4, pp. 439-465, 2002.

[41] F. Becattini, J. Cleymans, A. Keränen, E. Suhonen, and K. Redlich, "Features of particle multiplicities and strangeness production in central heavy ion collisions between $1.7 \mathrm{~A}$ and 158 A GeV/c," Physical Review C, vol. 64, no. 2, Article ID 024901, 2001.

[42] A. Keranen and F. Becattini, "The canonical effect in statistical models for relativistic heavy ion collisions," Journal of Physics G, vol. 28, p. 2041, 2002.

[43] A. Keranen and F. Becattini, "Chemical factors in canonical statistical models for relativistic heavy ion collisions," Physical Review C, vol. 65, Article ID 044901, 7 pages, 2002.

[44] J. Rafelski, J. Letessier, and A. Tounsi, "Strange particles from dense hadronic matter," Acta Physica Polonica B, vol. 27, pp. 1037-1140, 1996.

[45] K. Redlich and A. Tounsi, "Strangeness enhancement and energy dependence in heavy ion collisions," European Physical Journal C, vol. 24, no. 4, pp. 589-594, 2002.

[46] J. Sollfrank, P. Huovinen, M. Kataja, P. V. Ruuskanen, M. Prakash, and R. Venugopalan, "Hydrodynamical description of $200 \mathrm{~A} \mathrm{GeV/c} \mathrm{S+Au} \mathrm{collisions:} \mathrm{hadron} \mathrm{and} \mathrm{electromagnetic}$ spectra," Physical Review C, vol. 55, no. 1, pp. 392-410, 1997.

[47] P. Huovinena, P. F. Kolbb, U. Heinzb, P. V. Ruuskanend, and S. A. Voloshine, "Radial and elliptic flow at RHIC: further predictions," Physics Letters B, vol. 503, pp. 58-64, 2001.

[48] J. Cleymans and K. Redlich, "Chemical and thermal freeze-out parameters from $1 A$ to $200 \mathrm{~A} \mathrm{GeV,"} \mathrm{Physical} \mathrm{Review} \mathrm{C,} \mathrm{vol.} \mathrm{60,}$ Article ID 054908, 9 pages, 1999.

[49] J. Cleymans, H. Oeschler, and K. Redlich, "Influence of impact parameter on thermal description of relativistic heavy ion collisions at (1-2)A GeV," Physical Review C, vol. 59, no. 3, pp. 1663-1673, 1999.

[50] J. Cleymans, D. Elliott, A. Keränen, and E. Suhonen, “Thermal model analysis of particle ratios in $\mathrm{Ni}+\mathrm{Ni}$ experiments using exact strangeness conservation," Physical Review C, vol. 57, no. 6, pp. 3319-3323, 1998.

[51] J. Letessier and J. Rafelski, "Observing quark-gluon plasma with strange hadrons," International Journal of Modern Physics E, vol. 9, no. 2, pp. 107-147, 2000.
[52] P. Braun-Munzinger, J. Cleymans, H. Oeschler, and K. Redlich, "Maximum relative strangeness content in heavy-ion collisions around 30A GeV," Nuclear Physics A, vol. 697, no. 3-4, pp. 902912, 2002.

[53] W. Broniowski and W. Florkowski, "Description of the RHIC $\mathrm{p} \perp$ spectra in a thermal model with expansion," Physical Review Letters, vol. 87, no. 27, Article ID 272302, 2001.

[54] K. Redlich, "Strangeness production in heavy ion collisions," Nuclear Physics A, vol. 698, no. 1-4, p. 94, 2002.

[55] J. Cleymans and H. Satz, "Thermal hadron production in high energy heavy ion collisions," Zeitschrift für Physik C, vol. 57, no. 1, pp. 135-147, 1993.

[56] P. Braun-Munzinger and J. Stachel, "(Non) thermal aspects of charmonium production and a new look at $J / \psi$ suppression," Physics Letters B, vol. 490, no. 3-4, pp. 196-202, 2000.

[57] P. Braun-Munzinge and J. Stachel, "On charm production near the phase boundary," Nuclear Physics A, vol. 690, no. 1-3, p. 119, 2001.

[58] S. K. Tiwari, P. K. Srivastava, and C. P. Singh, "The effect of flow on hadronic spectra in an excluded-volume model," Journal of Physics G, vol. 40, Article ID 045102, 2013.

[59] G. M. Welke, R. Venugopalan, and M. Prakash, "The speed of sound in an interacting pion gas," Physics Letters B, vol. 245, no. 2, pp. 137-141, 1990.

[60] V. V. Dixit and E. Suhonen, "Bare-bones model for phase transition in hadronic matter," Zeitschrift für Physik C, vol. 18, no. 4, pp. 355-360, 1983.

[61] J. Cleymans, K. Redlich, H. Satz, and E. Suhonen, "On the phenomenology of deconfinement and chiral symmetry restoration," Zeitschrift für Physik C, vol. 33, no. 1, pp. 151-156, 1986.

[62] J. Cleymans, R. V. Gavai, and E. Suhonen, "Quarks and gluons at high temperatures and densities," Physics Reports, vol. 130, no. 4, pp. 217-292, 1986.

[63] J. Cleymans and E. Suhonen, "Influence of hadronic hard core radius on detonations and deflagrations in quark matter," Zeitschrift für Physik C, vol. 37, no. 1, pp. 51-56, 1987.

[64] J. Cleymans and D. W. Von Oertzen, "Bose-Einstein condensation of kaons in dense nuclear matter," Physics Letters B, vol. 249, no. 3-4, pp. 511-513, 1990.

[65] N. J. Davidson, H. G. Miller, R. M. Quick, and J. Cleymans, "Chemical equilibration in heavy-ion collisions," Physics Letters $B$, vol. 255, no. 1, pp. 105-109, 1991.

[66] H. Kuono and F. Takagi, "Excluded volume, bag constant and hadron-quark phase transition," Zeitschrift für Physik C, vol. 42, pp. 209-213, 1989.

[67] R. Hagedorn and J. Rafelski, "Hot hadronic matter and nuclear collisions," Physics Letters B, vol. 97, p. 136, 1980.

[68] R. Hagedorn, "The pressure ensemble as a tool for describing the hadron-quark phase transition," Zeitschrift für Physik C, vol. 17, pp. 265-281, 1983.

[69] D. H. Rischke, M. I. Gorenstein, H. Stöcker, and W. Greiner, "Excluded volume effect for the nuclear matter equation of state," Zeitschrift für Physik C, vol. 51, no. 3, pp. 485-489, 1991.

[70] C. P. Singh, B. K. Patra, and K. K. Singh, "Thermodynamically consistent EOS for hot dense hadron gas," Physics Letters B, vol. 387, no. 4, pp. 680-684, 1996.

[71] P. K. Panda, M. E. Bracco, M. Chiapparini, E. Conte, and G. Krein, "Excluded volume effects in the quark meson coupling model," Physical Review C, vol. 65, no. 6, Article ID 065206, 2002. 
[72] D. Anchishkin and E. Suhonen, "Generalization of mean-field models to account for effects of excluded volume," Nuclear Physics A, vol. 586, no. 4, pp. 734-754, 1995.

[73] D. Anchishkin, "Particle finite size effects as mean field approximation," Soviet Physics JETP, vol. 75, p. 195, 1992.

[74] N. Prasad, K. K. Singh, and C. P. Singh, "Causality in an excluded volume model," Physical Review C, vol. 62, no. 3, Article ID 037903, 2000.

[75] V. K. Tiwari, K. K. Singh, N. Prasad, and C. P. Singh, "Mean field model with excluded volume correction for a multicomponent hadron gas," Nuclear Physics A, vol. 637, no. 1, pp. 159-172, 1998.

[76] B. X. Sun, X. F. Lu, and E. G. Zhao, "Perturbative calculation of the excluded volume effect for nuclear matter in a relativistic mean-field approximation," Physical Review C, vol. 65, Article ID 054301, 2002.

[77] M. I. Gorenstein, "Examination of the thermodynamical consistency of excluded-volume hadron gas models," Physical Review C, vol. 86, Article ID 044907, 3 pages, 2012.

[78] Z.-D. Lu, A. Faessler, C. Fuchs, and E. E. Zabrodin, "Analysis of particle production in ultrarelativistic heavy-ion collisions within a two-source statistical model," Physical Review C, vol. 66, Article ID 044905, 7 pages, 2002.

[79] J. I. Kapusta, A. P. Vischer, and R. Venugopalan, "Nucleation of quark-gluon plasma from hadronic matter," Physical Review C, vol. 51, pp. 901-910, 1995.

[80] J. I. Kapusta and K. A. Olive, "Thermodynamics of hadrons: delimiting the temperature," Nuclear Physics A, vol. 408, no. 3, pp. 478-494, 1983.

[81] J. D. Walecka, "A theory of highly condensed matter," Annals of Physics, vol. 83, no. 2, pp. 491-529, 1974.

[82] B. D. Serot and J. D. Walecka, "Properties of finite nuclei in a relativistic quantum field theory," Physics Letters B, vol. 87, no. 3, pp. 172-176, 1979.

[83] D. H. Rischke, B. L. Friman, H. Stocker, and W. Greiner, "Phase transition from hadron gas to quark-gluon plasma: influence of the stiffness of the nuclear equation of state," Journal of Physics G, vol. 14, no. 2, pp. 191-203, 1988.

[84] J. Zimányi, B. Lukács, P. Lévai, J. P. Bondorf, and N. L. Balazs, "An interpretable family of equations of state for dense hadronic matter," Nuclear Physics A, vol. 484, no. 3-4, pp. 647-660, 1988.

[85] K. A. Bugaev and M. I. Gorenstein, “Thermodynamically selfconsistent class of nuclear matter EOS and compression shocks in relativistic nuclear collisions," Zeitschrift für Physik C, vol. 43, no. 2, pp. 261-265, 1989.

[86] G. D. Yen, M. I. Gorenstein, W. Greiner, and S. N. Yang, "Excluded volume hadron gas model for particle number ratios in a+a collisions," Physical Review C, vol. 56, no. 4, pp. $2210-$ 2218, 1997.

[87] F. Becattini, "A Thermodynamical approach to hadron production in e+ e- collisions," Zeitschrift für Physik C, vol. 69, pp. 485492, 1996.

[88] F. Becattini and U. Heinz, "Thermal hadron production in pp and ppc̄ollisions," Zeitschrift für Physik C, vol. 76, no. 2, pp. 269286, 1997.

[89] S. Uddin and C. P. Singh, "Equation of state of finite-size hadrons: thermodynamical consistency," Zeitschrift für Physik C, vol. 63, no. 1, pp. 147-150, 1994.

[90] C. P. Singh, P. K. Srivastava, and S. K. Tiwari, "QCD phase boundary and critical point in a bag model calculation," Physical Review D, vol. 80, Article ID 114508, 2011.
[91] C. P. Singh, P. K. Srivastava, and S. K. Tiwari, "Erratum in QCD phase boundary and critical point in a bag model calculation," Physical Review D, vol. 83, Article ID 039904, 1 page, 2011.

[92] G. Zeeb, K. A. Bugaev, P. T. Reuter, and H. Stöcker, "Equation of state for the two-component van der waals gas with relativistic excluded volumes," Ukrainian Journal of Physics, vol. 53, no. 3, pp. 279-295, 2008.

[93] K. A. Bugaev, Ph.D. thesis, ch 3.

[94] F. Becattini, M. Gazdzicki, A. Keranen, J. Manninen, and R. Stock, "Chemical equilibrium study in nucleus-nucleus collisions at relativistic energies," Physical Review C, vol. 69, Article ID 024905, 19 pages, 2004.

[95] J. Letessier and J. Rafelski, "Hadron production and phase changes in relativistic heavy-ion collisions," The European Physical Journal A, vol. 35, pp. 221-242, 2008.

[96] A. Dumitru, L. Portugal, and D. Zschiesche, "Inhomogeneous freeze-out in relativistic heavy-ion collisions," Physical Review C, vol. 73, no. 2, Article ID 024902, 2006.

[97] F. Becattini and G. Passaleva, "Statistical hadronization model and transverse momentum spectra of hadrons in high energy collisions," European Physical Journal C, vol. 23, no. 3, pp. 551$583,2002$.

[98] M. Kaneta and N. Xu, "Centrality dependence of chemical freeze-out in Au+Au collisions at RHIC," http://arxiv.org/abs/ nucl-th/0405068.

[99] J. Adams and STAR Collaboration, "Experimental and theoretical challenges in the search for the quark gluon plasma. The STAR Collaboration's Critical Assessment of the Evidence from RHIC Collisions," Nuclear Physics A, vol. 757, p. 102, 2005.

[100] A. Tawfik, "Chemical freeze-out and higher order multiplicity moments," http://arxiv.org/abs/1306.1025.

[101] S. Gupta, X. Luo, B. Mohanty, H. G. Ritter, and N. Xu, "Scale for the phase diagram of quantum chromodynamics," Science, vol. 332, no. 6037, pp. 1525-1528, 2011.

[102] A. Bazavov, H.-T. Ding, and P. Hegde, "Freeze-out conditions in heavy ion collisions from QCD thermodynamics," Physical Review Letters, vol. 109, Article ID 192302, 5 pages, 2012.

[103] J. Cleymans, H. Oeschler, K. Redlich, and S. Wheaton, "Comparison of chemical freeze-out criteria in heavy-ion collisions," Physical Review C, vol. 73, no. 3, Article ID 034905, 2006.

[104] C. Alt and NA49 Collaboration, "Pion and kaon production in central $\mathrm{Pb}+\mathrm{Pb}$ collisions at $20 \mathrm{~A}$ and $30 \mathrm{~A} \mathrm{GeV}$ : evidence for the onset of deconfinement," Physical Review C, vol. 77, Article ID 024903, 10 pages, 2008.

[105] M. Gazdzicki and the NA49 Collaboration, "Report from NA49," Journal of Physics G, vol. 30, p. 701, 2004.

[106] S. V. Afanasiev, T. Anticic, and D. Barna, "Energy dependence of pion and kaon production in central $\mathrm{Pb}+\mathrm{Pb}$ collisions," Physical Review C, vol. 66, Article ID 054902, 2002.

[107] T. Anticic, B. Baatar, D. Barna et al., " $\Lambda$ and $\bar{\Lambda}$ production in central $\mathrm{Pb}-\mathrm{Pb}$ collisions at 40,80 , and $158 \mathrm{~A} \mathrm{GeV,"} \mathrm{Physical}$ Review Letters, vol. 93, Article ID 022302, 2004.

[108] L. Ahle, L. Ahle, Y. Akiba, and K. Ashktorab, "Particle production at high baryon density in central $\mathrm{Au}+\mathrm{Au}$ reactions at $11.6 \mathrm{~A}$ GeV/c," Physical Review C, vol. 57, pp. R466-R470, 1998.

[109] L. Ahle, Y. Akiba, K. Ashktorab et al., "Centrality dependence of kaon yields in $\mathrm{Si}+\mathrm{A}$ and $\mathrm{Au}+\mathrm{Au}$ collisions at the AGS," Physical Review C, vol. 60, Article ID 044904, 1999.

[110] S. Albergo, R. Bellwied, and M. Bennett, " $\Lambda$ spectra in $11.6 \mathrm{~A}$ GeV/c Au-Au collisions," Physical Review Lett, vol. 88, Article ID 062301, 2002. 
[111] J. L. Klay, N. N. Ajitanand, J. M. Alexander et al., "Charged pion production in $2 A$ to $8 A \mathrm{GeV}$ central $\mathrm{Au}+\mathrm{Au}$ collisions," Physical Review C, vol. 68, Article ID 054905, 2003.

[112] S. Chatterjee, R. M. Godbole, and S. Gupta, "Stabilizing hadron resonance gas models," Physical Review C, vol. 81, no. 4, Article ID 044907, 2010.

[113] R. Stock, "Relativistic nucleus-nucleus collisions: from the BEVALAC to RHIC," Journal of Physics G, vol. 30, no. 8, pp. S633-S648, 2004.

[114] J. Noronha-Hostler, H. Ahmad, J. Noronha, and C. Greiner, "Particle ratios as a probe of the QCD critical temperature," Physical Review C, vol. 82, no. 2, Article ID 024913, 2010.

[115] D. Molnar and S. A. Voloshin, "Elliptic flow at large transverse momenta from quark coalescence," Physical Review Letters, vol. 91, Article ID 092301, 2003.

[116] D. Molnar, "Particle correlations at RHIC from parton coalescence dynamics-first results," Journal of Physics G, vol. 30, p. S1239, 2004.

[117] C. Alt, T. Anticic, and B. Baatar, "Energy dependence of $\Lambda$ and $\equiv$ production in central $\mathrm{Pb}+\mathrm{Pb}$ collisions at $20 \mathrm{~A}, 30 \mathrm{~A}$, $40 A, 80 A$, and $158 A \mathrm{GeV}$ measured at the CERN Super Proton Synchrotron," Physical Review C, vol. 78, Article ID 034918, 2008.

[118] C. Blume and The NA49 collaboration, "Review of results from the NA49 Collaboration," Journal of Physics G, vol. 31, no. 6, pp. S685-S691, 2005.

[119] M. M. Aggarwal and The STAR Collaboration, "Strange and multi-strange particle production in $\mathrm{Au}+\mathrm{Au}$ collisions at $\sqrt{\mathrm{sNN}}$ = 62.4 GeV," Physical Review C, vol. 83, Article ID 024901, 2011.

[120] A. Andronic, D. Blaschke, P. Braun-Munzinger et al., "Hadron production in ultra-relativistic nuclear collisions: quarkyonic matter and a triple point in the phase diagram of QCD," Nuclear Physics A, vol. 837, no. 1-2, pp. 65-86, 2010.

[121] B. Abelev, N. Arbor, G. Conesa Balbastre et al., "Pion, Kaon, and Proton production in central $\mathrm{Pb}-\mathrm{Pb}$ Collisions at $\sqrt{s_{N N}}=2.76$ TeV," Physical Review Letters, vol. 109, Article ID 252301, 2012.

[122] N. Sasaki, "A study of the thermodynamic properties of a hot, dense hadron gas using an event generator: hadro-moleculardynamical calculation," Progress of Theoretical Physics, vol. 10, no. 4, pp. 783-805, 2001.

[123] P. Braun-Munzinger and J. Stachel, "Particle ratios, equilibration and the QCD phase boundary," Journal of Physics G, vol. 28, no. 7, pp. 1971-1976, 2002.

[124] V. Magas and H. Satz, "Conditions for confinement and freezeout," European Physical Journal C, vol. 32, no. 1, pp. 115-119, 2003.

[125] A. Tawfik, "Influence of strange quarks on the QCD phase diagram and chemical freeze-out," Journal of Physics G, vol. 31, no. 6, pp. S1105-S1110, 2005.

[126] M. B. Isichenko, "Percolation, statistical topography, and transport in random media," Reviews of Modern Physics, vol. 64, no. 4, pp. 961-1043, 1992.

[127] J. Kapusta, "Viscous heating of expanding fireballs," Physical Review C, vol. 24, no. 6, pp. 2545-2551, 1981.

[128] A. M. Polyakov, Journal of Experimental and Theoretical Physics, vol. 57, p. 2144, 1969.

[129] F. Karsch, D. Kharzeev, and K. Tuchin, "Universal properties of bulk viscosity near the QCD phase transition," Physics Letters B, vol. 663 , no. 3, pp. 217-221, 2008.

[130] U. Heinz and P. Kolb, "Early thermalization at RHIC," Nuclear Physics A, vol. 702, no. 1-4, p. 269, 2002.
[131] A. Adare and PHENIX Collaboration, "Energy loss and flow of heavy quarks in $\mathrm{Au}+\mathrm{Au}$ collisions at $\sqrt{\mathrm{sNN}}=200 \mathrm{GeV}$," Physical Review Letters, vol. 98, Article ID 172301, 2007.

[132] E. Shuryak, "What RHIC experiments and theory tell us about properties of quark-gluon plasma?” Nuclear Physics A, vol. 750, no. 1, pp. 64-83, 2005.

[133] P. Romatschke and U. Romatschke, "Viscosity information from relativistic nuclear collisions: how perfect is the fluid observed at RHIC?” Physical Review Letters, vol. 99, no. 17, Article ID 172301, 2007.

[134] S. Gavin, "Transport coefficients in ultra-relativistic heavy-ion collisions," Nuclear Physics A, vol. 435, no. 3-4, pp. 826-843, 1985.

[135] A. Dobado and S. N. Santalla, "Pion gas viscosity at low temperature and density," Physical Review D, vol. 65, no. 9, Article ID 096011, 2002.

[136] M. Prakash, M. Prakash, R. Venugopalan, and G. Welke, "Nonequilibrium properties of hadronic mixtures," Physics Report, vol. 227, no. 6, pp. 321-366, 1993.

[137] J.-W. Chen, Y.-H. Li, Y.-F. Liu, and E. Nakano, "QCD viscosity to entropy density ratio in the hadronic phase," Physical Review $D$, vol. 76, Article ID 114011, 8 pages, 2007.

[138] J.-W. Chen and E. Nakano, "Shear viscosity to entropy density ratio of QCD below the deconfinement temperature," Physics Letters B, vol. 647, pp. 371-375, 2007.

[139] K. Itakura, O. Morimatsu, and H. Otomo, "Shear viscosity of a hadronic gas mixture," Physical Review D, vol. 77, no. 1, Article ID 014014, 2008.

[140] A. S. Khvorostukhin, V. D. Toneev, and D. N. Voskresensky, "Viscosity coefficients for hadron and quark-gluon phases," Nuclear Physics A, vol. 845, no. 1-4, pp. 106-146, 2010.

[141] A. Muronga, "Shear viscosity coefficient from microscopic models," Physical Review C, vol. 69, no. 4, Article ID 044901, 2004.

[142] S. Muroya and N. Sasaki, "A calculation of the viscosity to entropy ratio of a hadronic gas," Progress of Theoretical Physics, vol. 113, no. 2, pp. 457-462, 2005.

[143] N. Demir and S. A. Bass, "Shear-viscosity to entropy density ratio of a relativistic hadron gas," Physical Review Letters, vol. 102, Article ID 172302, 2009.

[144] L. P. Csernai, J. I. Kapusta, and L. D. McLerran, "Strongly interacting low-viscosity matter created in relativistic nuclear collisions," Physical Review Letters, vol. 97, no. 15, Article ID 152303, 2006.

[145] J. Noronha-Hostler, J. Noronha, and C. Greiner, "Transport coefficients of hadronic matter near Tc," Physical Review Letters, vol. 103, no. 17, Article ID 172302, 2009.

[146] R. A. Lacey, N. N. Ajitanand, J. M. Alexander et al., "Has the QCD critical point been signaled by observations at the BNL relativistic heavy ion collider?" Physical Review Letters, vol. 98, Article ID 092301, 2007.

[147] A. Dobado, F. J. Llanes-Estrada, and J. M. Torres-Rincon, " $\eta / \mathrm{s}$ and phase transitions," Physical Review D, vol. 79, no. 1, Article ID 014002, 2009.

[148] A. Dobado, F. Llanes-Estrada, and J. M. Torres-Rincon, "eta/s and phase transitions," Physical Review D, vol. 79, Article ID 014002, 2009.

[149] S. Pal, "Shear viscosity to entropy density ratio of a relativistic Hagedorn resonance gas," Physics Letters B, vol. 684, pp. 211-215, 2010. 
[150] P. Castorina, J. Cleymans, D. E. Miller, and H. Satz, “The speed of sound in hadronic matter," European Physical Journal C, vol. 66, no. 1, pp. 207-213, 2010.

[151] J. Cleymans and D. Worku, “The Hagedorn temperature revisited," Modern Physics Letters A, vol. 26, no. 16, pp. 1197-1209, 2011.

[152] R. V. Gavai and A. Gocksch, "Velocity of sound in SU(3) lattice gauge theory," Physical Review D, vol. 33, no. 2, pp. 614-616, 1986.

[153] K. Redlich and H. Satz, "Critical behavior near deconfinement," Physical Review D, vol. 33, no. 12, pp. 3747-3752, 1986.

[154] F. Karsch, "Recent lattice results on finite temperature and density QCD-part I," Proceedings of Science, CPOD07, 026, 2007.

[155] D. Prorok and L. Turko, " $J / \psi$ absorption in a multicomponent hadron gas," in Proceedings of the AIP Conference, vol. 1038, p. $10,2008$.

[156] M. Chojnacki and W. Florkowski, “Temperature dependence of sound velocity and hydrodynamics of ultra-relativistic heavyion collisions," Acta Physica Polonica B, vol. 38, pp. 3249-3262, 2007.

[157] M. I. Gorenstein, M. Hauer, and O. N. Moroz, "Viscosity in the excluded volume hadron gas model," Physical Review C, vol. 77, no. 2, Article ID 024911, 2008.

[158] E. M. Lifschitz and L. P. Pitaevski, Physical Kinetics, Pergamon Press, Oxford, UK, 2nd edition, 1981.

[159] G. Policastro, D. T. Son, and A. O. Starinets, "Shear viscosity of strongly coupled $\mathrm{N}=4$ supersymmetric Yang-Mills plasma," Physical Review Letters, vol. 87, no. 8, Article ID 081601, 2001.

[160] J. M. Maldacena, "The large N limit of superconformal field theories and supergravity," Advances in Theoretical and Mathematical Physics, vol. 2, pp. 231-252, 1998.

[161] Z. Qiu, C. Shen, and U. Heinz, "Hydrodynamic elliptic and triangular flow in $\mathrm{Pb}-\mathrm{Pb}$ collisions at $\sqrt{s_{N N}}=2.76 \mathrm{~A} \mathrm{TeV}$," Physics Letters B, vol. 707, no. 1, pp. 151-155, 2012.

[162] J. Letessier and J. Rafelski, Hadrons and Quark-Gluon Plasma, Cambridge University Press, Cambridge, UK, 2004.

[163] L. D. Landau, Izvestiya Akademii Nauk, vol. 17, p. 51, 1953.

[164] E. Schnedermann, J. Sollfrank, and U. Heinz, "Thermal phenomenology of hadrons from $200 \mathrm{~A} \mathrm{GeV} \mathrm{S+S} \mathrm{collisions,"} \mathrm{Physi-}$ cal Review C, vol. 48, no. 5, pp. 2462-2475, 1993.

[165] E. Schnedermann and U. Heinz, "Circumstantial evidence for transverse flow in $200 \mathrm{~A} \mathrm{GeV} \mathrm{S+S} \mathrm{collisions,"} \mathrm{Physical} \mathrm{Review}$ Letters, vol. 69, no. 20, pp. 2908-2911, 1992.

[166] S. Q. Feng and Y. Zhong, "Baryon production and collective flow in relativistic heavy-ion collisions in the AGS, SPS, RHIC, and LHC energy regions $\left(\sqrt{s_{N N}} \leq 5 \mathrm{GeV}\right.$ to $\left.5.5 \mathrm{TeV}\right)$," Physical Review C, vol. 83, Article ID 034908, 2011.

[167] S. Q. Feng and X. B. Yuan, "The feature study on the pion and proton rapidity distributions at AGS, SPS and RHIC," Science in China G, vol. 52, p. 198, 2009.

[168] S. Uddin, J. S. Ahmad, W. Bashir, and R. A. Bhat, "A unified approach towards describing rapidity and transverse momentum distributions in a thermal freeze-out model," Journal of Physics G, vol. 39, Article ID 015012, 2012.

[169] T. Hirano, K. Morita, S. Muroya, and C. Nonaka, "Hydrodynamical analysis of hadronic spectra in the $130 \mathrm{GeV} /$ nucleon $\mathrm{Au}+\mathrm{Au}$ collisions," Physical Review C, vol. 65, no. 6, Article ID 061902, 2002.
[170] K. Morita, S. Muroya, C. Nonaka, and T. Hirano, "Comparison of space-time evolutions of hot, dense matter in $\sqrt{s_{N N}}=$ 17 and $130 \mathrm{GeV}$ relativistic heavy ion collisions based on a hydrodynamical model," Physical Review C, vol. 66, Article ID 054904, 2002.

[171] J. Manninen, E. L. Bratkovskaya, W. Cassing, and O. Linnyk, "Dilepton production in $p+p, \mathrm{Cu}+\mathrm{Cu}$ and $\mathrm{Au}+\mathrm{Au}$ collisions at $\sqrt{\mathrm{s}}=200 \mathrm{GeV}$,' European Physical Journal C, vol. 71, article 1615, 2011.

[172] S. A. Bass, M. Belkacem, and M. Bleicher, "Microscopic models for ultrarelativistic heavy ion collisions," Progress in Particle and Nuclear Physics, vol. 41, pp. 255-369, 1998.

[173] U. Mayer and U. Heinz, "Global hydrodynamics with continuous freeze-out," Physical Review C, vol. 56, no. 1, pp. 439-452, 1997.

[174] U. Heinz, "Primordial hadrosynthesis in the little bang," Nuclear Physics A, vol. 661, pp. 140-149, 1999.

[175] F. Becattini and J. Cleymans, "Chemical equilibrium in heavy ion collisions: rapidity dependence," Journal of Physics G, vol. 34, no. 8, pp. S959-S963, 2007.

[176] F. Becattini, J. Cleymans, and J. Strumpfer, "Rapidity variation of thermal parameters at SPS and RHIC," Proceeding of Science, CPOD07, 012, 2007.

[177] B. Biedron and W. Broniowski, "Rapidity-dependent spectra from a single-freeze-out model of relativistic heavy-ion collisions," Physical Review C, vol. 75, Article ID 054905, 2007.

[178] W. Broniowski and B. Biedron, "Rapidity-dependent chemical potentials in a statistical approach," Journal of Physics G, vol. 35, Article ID 044018, 2008.

[179] J. Cleymans, J. Strümpfer, and L. Turko, "Extended longitudinal scaling and the thermal model," Physical Review C, vol. 78, no. 1, Article ID 017901, 2008.

[180] W. Broniowski and W. Florkowski, "Geometric relation between centrality and the impact parameter in relativistic heavy-ion collisions," Physical Review C, vol. 65, no. 2, Article ID 064905, 2002.

[181] C. Adler, Z. Ahammed, and C. Allgower, "Measurement of inclusive antiprotons from $\mathrm{Au}+\mathrm{Au}$ collisions at $\sqrt{s_{N N}}=130$ GeV," Physical Review Letters, vol. 87, Article ID 262302, 2001.

[182] K. Adcox, S. S. Adler, N. N. Ajitanand et al., "Single identified hadron spectra from $\sqrt{s_{N N}}=130 \mathrm{GeV} \mathrm{Au+Au} \mathrm{collisions,"}$ Physical Review C, vol. 69, Article ID 024904, 2004.

[183] A. Adare, C. Aidala, and N. N. Ajitanand, "Cold-nuclearmatter effects on heavy-quark production in $d+A u$ collisions at $\sqrt{s_{N N}}=200 \mathrm{GeV}$," Physical Review Letters, vol. 109, Article ID 242301, 7 pages, 2012.

[184] P. Bozek, "Viscous evolution of the rapidity distribution of matter created in relativistic heavy-ion collisions," Physical Review C, vol. 77, Article ID 034911, 2008.

[185] Y. B. Ivanov, Physical Review C, vol. 87, Article ID 064905, 2013.

[186] P. Huovinen, P. F. Kolb, and U. Heinz, "Is there elliptic flow without transverse flow?" Nuclear Physics A, vol. 698, no. 1-4, p. $475,2002$.

[187] Y. B. Ivanov, V. N. Russkikh, and V. D. Toneev, "Relativistic heavy-ion collisions within three-fluid hydrodynamics: hadronic scenario," Physical Review C, vol. 73, no. 4, Article ID 044904, 2006.

[188] B. Mohanty and J. Alam, "Velocity of sound in relativistic heavyion collisions," Physical Review C, vol. 68, Article ID 064903, 2003. 
[189] P. K. Netrakanti and B. Mohanty, "Width of the rapidity distribution in heavy-ion collisions," Physical Review C, vol. 71, no. 4, Article ID 047901, 2005.

[190] F. Cooper and G. Frye, "Single-particle distribution in the hydrodynamic and statistical thermodynamic models of multiparticle production," Physical Review D, vol. 10, no. 1, pp. 186189, 1974.

[191] D. Adamová, G. Agakichiev, H. Appelshäuser et al., "Universal pion freeze-out in heavy-ion collisions," Physical Review Letters, vol. 90, Article ID 022301, 2003.

[192] I. G. Bearden, "Charged meson rapidity distributions in central $\mathrm{Au}+\mathrm{Au}$ collisions at $\sqrt{s_{N N}}=200 \mathrm{GeV}$," Physical Review Letters, vol. 94, Article ID 162301, 2005.

[193] S. S. Adler, S. Afanasiev, and C. Aidala, "Identified charged particle spectra and yields in $\mathrm{Au}+\mathrm{Au}$ collisions at $\sqrt{s_{N N}}=200$ GeV," Physical Review C, vol. 69, Article ID 034909, 2004.

[194] M. Floris and ALICE collaboration, "Identified particles in pp and $\mathrm{Pb}-\mathrm{Pb}$ collisions at LHC energies with the ALICE detector," Journal of Physics G, vol. 38, Article ID 124025, 2011.

[195] C. Shen, U. Heinz, P. Huovinen, and H. Song, "Radial and elliptic flow in $\mathrm{Pb}+\mathrm{Pb}$ collisions at energies available at the CERN Large Hadron Collider from viscous hydrodynamics," Physical Review C, vol. 84, no. 4, Article ID 044903, 2011.

[196] P. K. Srivastava and C. P. Singh, "A hybrid model for QCD deconfining phase boundary," Physical Review D, vol. 85, Article ID 114016, 2012.

[197] P. K. Srivastava, S. K. Tiwari, and C. P. Singh, "QCD critical point in a quasiparticle model," Physical Review D, vol. 82, no. 1, Article ID 014023, 2010.

[198] P. K. Srivastava, S. K. Tiwari, and C. P. Singh, "On locating the critical end point in QCD phase diagram," Nuclear Physics A, vol. 862-863, pp. 424-426, 2011. 

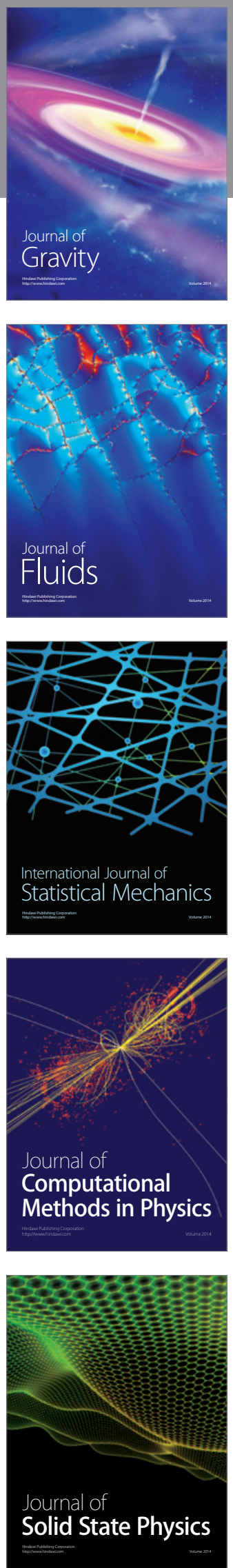

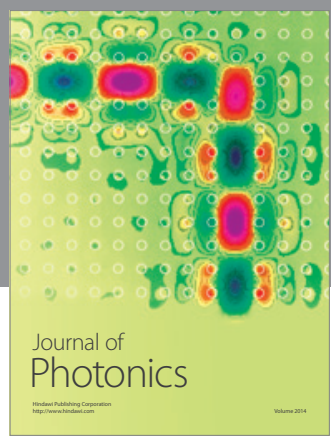

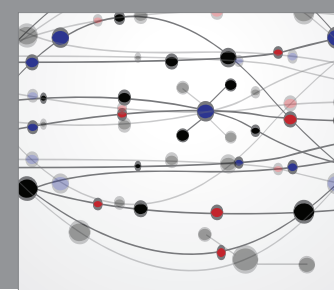

The Scientific World Journal

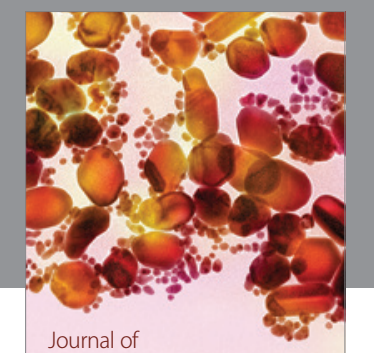

Soft Matter
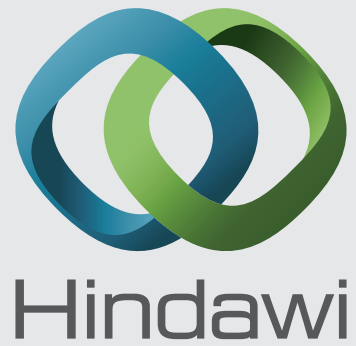

Submit your manuscripts at

http://www.hindawi.com
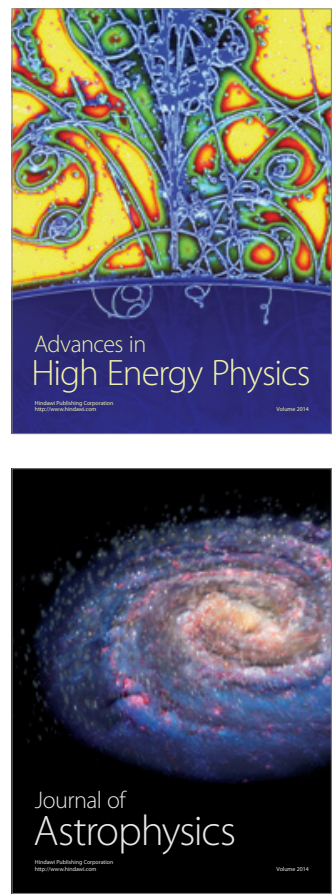
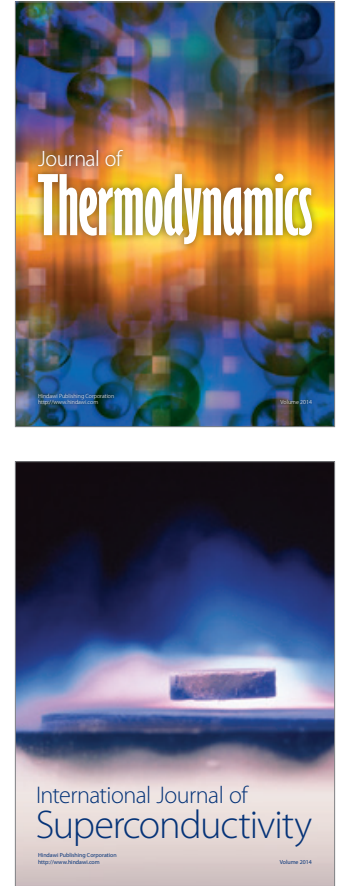
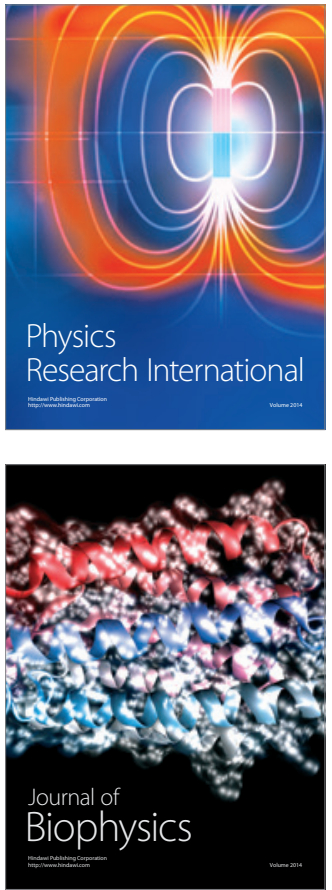
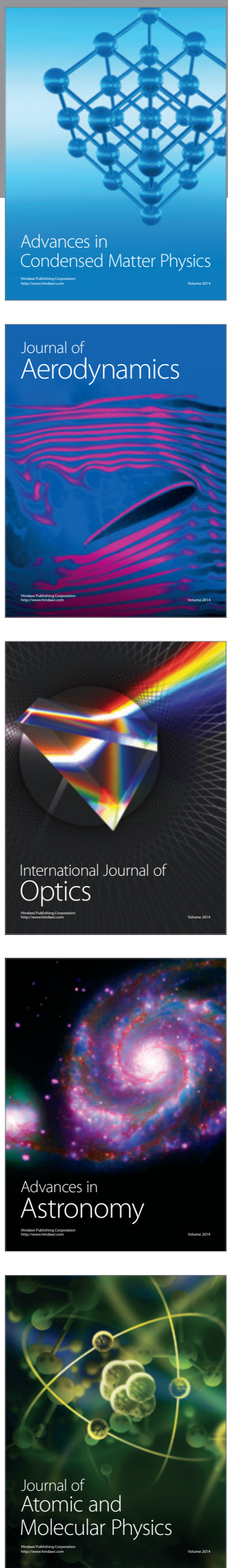